\title{
A New Look for the Red Macroalga Palmaria palmata: A Seafood with Polar Lipids Rich in EPA and with Antioxidant Properties
}

\author{
Diana Lopes ${ }^{1,2}$, Tânia Melo ${ }^{1,2}$, Joana Meneses ${ }^{1}$, Maria H. Abreu ${ }^{3}$, Rui Pereira ${ }^{3}$, \\ Pedro Domingues ${ }^{1}\left(\mathbb{D}\right.$, Ana I. Lillebø ${ }^{4}$, Ricardo Calado ${ }^{4}$ (D) and M. Rosário Domingues $1,2, *(\mathbb{D})$ \\ 1 Centro de Espectrometria de Massa, Departamento de Química \& QOPNA-LAQV, Universidade de Aveiro, \\ Campus Universitário de Santiago, 3810-193 Aveiro, Portugal \\ 2 Departamento de Química \& CESAM \& ECOMARE, Universidade de Aveiro, Campus Universitário de \\ Santiago, 3810-193 Aveiro, Portugal \\ 3 ALGAplus-Produção e Comercialização de algas e seus derivados, Lda., 3830-352 Ílhavo, Portugal \\ 4 Departamento de Biologia \& CESAM \& ECOMARE, Universidade de Aveiro, Campus Universitário de \\ Santiago, 3810-193 Aveiro, Portugal \\ * Correspondence: mrd@ua.pt; Tel.: +351-234-401-505
}

Received: 29 July 2019; Accepted: 11 September 2019; Published: 13 September 2019

\begin{abstract}
Palmaria palmata is an edible red macroalga widely used for human consumption and valued for its high protein value. Despite its low total lipid content, it is rich in eicosapentaenoic acid (EPA). This seaweed has been scarcely explored with regard to its lipid composition. The polar lipids of seaweeds are nowadays recognized as important phytochemicals contributing to their add value valorization and providing support for claims of potential health benefits. The present study aimed to disclose the polar lipid profile of P. palmata, farmed in an integrated multi-trophic aquaculture (IMTA) through modern lipidomic approaches using high-resolution LC-MS and MS/MS and to screen for the antioxidant properties of this red macroalga. A total of 143 molecular species of lipids were identified, belonging to several classes of polar lipids, such as glycolipids, phospholipids, and betaine lipids. It is noteworthy that the most abundant lipid species in each class were esterified with eicosapentaenoic acid (EPA), accounting for more than 50\% of the lipid content. The polar lipid extract rich in EPA showed antioxidant activity with an inhibition concentration (IC) of IC30 = 171 $\pm 19.8 \mu \mathrm{g} / \mathrm{mL}$ for $\alpha, \alpha$-diphenyl- $\beta$-picrylhydrazyl radical $\left(\mathrm{DPPH}^{\bullet}\right)$ and IC50 = $26.2 \pm 0.1 \mu \mathrm{g} / \mathrm{mL}$ for 2,20-azino-bis-3-ethylbenzothiazoline-6-sulfonic acid radical cation (ABTS ${ }^{\bullet+}$ ). Overall, this study highlights that P. palmata farmed in an IMTA framework can be a sustainable source of beneficial lipids with antioxidant activity. Moreover, this red macroalga can be exploited for future applications as a source of lipids rich in EPA for food and feed, nutraceuticals, and cosmetics.
\end{abstract}

Keywords: IMTA; lipidome; lipidomics; mass spectrometry; rhodophyta; seaweeds

\section{Introduction}

Over the last 20 years, macroalgae have ceased to be a trifle in western countries and have been considered as a nutritional and functional food, generating wealth for many coastal countries [1]. Red macroalgae (phylum Rhodophyta) are mainly used as a source of hydrocolloids (agar and carrageenans), generating a large turnover worldwide [2]. Nevertheless, the marketing of edible red macroalgae is also very important, as is the trade of Asian species of Porphyra sp., the most valued variety of seaweed in the world, generally recognized by its common Japanese name, nori. Recently, some of the species of the genus Porphyra were transferred to the genus Pyropia [3]. Food and agriculture organization of the united nations (FAO) data indicate that the production of Porphyra 
sp. is global, but Japan, China, and the Republic of Korea are still the main producers [4]. From 2000 to 2016, the Global Aquaculture Production for Porphyra sp. increased from 424,913 to 1,352,520 tons, demonstrating the enormous demand for these edible red seaweeds [4]. Nonetheless, there is another valuable red macroalga that has remained largely overlooked, Palmaria palmata ((Linnaeus) F. Weber \& D. Mohr, 1805), also known as dulse (Palmariales, Rhodophyta). It is an edible red macroalga found in the coastal waters of the North Atlantic [5]. Its lipid content, as in most macroalgae, compared to other photosynthetic lipid sources is considered low (0.3-3.8\% of dry weight) and erroneously devalued [6]. However, the lipids of P. palmata are easily assimilated by the human body and have a high content of eicosapentaenoic acid (EPA), a golden fatty acid (FA) in healthy diets and important in the prevention of non-communicable diseases (NCDs), which are responsible for the deaths of 41 million people each year (equivalent to $71 \%$ of all deaths worldwide) [7,8]. Eicosapentaenoic acid is an omega-3 (n-3) polyunsaturated fatty acid(PUFA) that plays an effective role in the improvement and prevention of cardiovascular and neurodegenerative diseases, as well as beneficial antioxidant and anti-inflammatory effects [9-11]. The PUFA of seaweeds are generally located in the structural lipids found in their membranes in the form of phospholipids (PLs) and glycolipids (GLs). To our best knowledge, the polar lipids of Palmaria palmata have not been studied so far, and the lack of knowledge on this subject must be overcome because polar lipids bearing EPA have recently been associated with several bioactive properties, such as antitumor [12,13], anti-inflammatory [14,15], antimicrobial [16,17], and antiviral [18]. Thus, the identification of the P. palmata lipidome is crucial for future exploitation and valorization. In addition, seaweed lipids are also recognized as natural and organic antioxidants with different applications [19]. Today, there is an increasing demand for new antioxidants for the food, feed, nutraceuticals, and cosmetic industry, and consequently, an increasing bioprospecting effort. Food containing phytochemical antioxidants can be used as nutritional supplements and functional foods and to extend the shelf life of food for human consumption. In addition, the cosmetic industry is increasingly looking for natural antioxidants recognized as active ingredients for cosmetic formulations that alter the effects of ageing and act as inhibitors of oxidants [20]. Nevertheless, to our best knowledge, the lipid antioxidant potential of P. palmata has not yet been explored.

Knowing the lipid profile of macroalgae can help to reveal their healthy attributes and their added value. The use of lipidomics approaches based on liquid chromatography mass spectrometry (LC-MS) has been successfully used for this purpose [21-25] but has not yet been applied to the profiling of $P$. palmata. The present study characterized the polar lipid signature of $P$. palmata farmed in an integrated multi-trophic aquaculture (IMTA) system. A lipidomic approach based on LC-MS was used to detect lipids bearing $n-3$ fatty acids. Also, the antioxidant activity of the polar lipid-rich extract was also screened, through the free radical scavenging potential against $\alpha, \alpha$-diphenyl- $\beta$-picrylhydrazyl (DPPH) and 2,20-azino-bis-3-ethylbenzothiazoline-6-sulfonic acid (ABTS) radicals. Our goal was to add value to P. palmata by presenting it as a bioactive lipid source and EPA carrier with potential applications in the fields of food, feed, pharma, and cosmetics.

\section{Results}

\subsection{Identification of the of Palmaria Palmata Lipidome}

The lipid extracts of P. palmata represented an average yield of $1 \%$ by dry weight $(255.6 \pm 1.19 \mathrm{mg}$ of biomass, $2.58 \pm 0.19 \mathrm{mg}$ lipid extract). The fatty acid composition of the lipid extract of P. palmata, identified by gas chromatography-mass spectrometry (GC-MS) (Table 1), shows the prevalence of 20:5(n-3) EPA with a relative abundance of $51.68 \% \pm 6.5$. The saturated FA 16:0, 18:0, and 14:0 represented $24.32 \% \pm 1.11,12.45 \% \pm 6.74$, and $5.32 \pm 0.44$, respectively. The others FA identified had relative abundances of less than 5\%. The GC-MS analysis also identified phytol compounds, a component of chlorophyll and vitamin E and K. Phytol is a precursor of vitamin E, an important nutritional supplement obtained commercially by isolation from natural sources [26]. 
Table 1. Fatty acid profile of $P$. palmata determined by GC-MS analysis. Abundances are expressed in relative abundance $(\%)$. Values are averages of five samples \pm standard deviation. Double bond position of 18:1 and 18:2 was not identified.

\begin{tabular}{cc}
\hline Fatty Acid & Relative Abundance (\%) \pm SD \\
\hline $14: 0$ & $5.32 \pm 0.44$ \\
$16: 0$ & $24.32 \pm 1.11$ \\
$16: 1(n-7)$ & $2.03 \pm 0.43$ \\
$18: 0$ & $12.45 \pm 6.74$ \\
$18: 1$ & $2.82 \pm 0.54$ \\
$18: 2$ & $0.45 \pm 0.19$ \\
$20: 4(n-6)$ & $0.92 \pm 0.17$ \\
$20: 5(n-3)$ & $51.68 \pm 6.47$ \\
\hline
\end{tabular}

Identification of the polar lipid profile at the molecular level was performed by high-resolution hydrophilic interaction liquid chromatography-mass spectrometry - HILIC-LC-MS and HILIC-LC-MS/MS. In total, 46 molecular species of glycolipids were identified, 1 molecular species of betaine lipids, 91 molecular species of phospholipids, and 6 molecular species of inositephosphoceramide lipids, representing a total of 144 species of lipids. The glycolipids identified included the acidic glycolipids sulfoquinovosyl diacylglycerol (SQDG) (Figure 1) and sulfoquinovosyl monoacylglycerol (SQMG) classes, assigned as $[\mathrm{M}-\mathrm{H}]^{-}$ions in the LC-MS spectra (Table 2) and the neutral glycolipids monogalactosyldiacylglycerol (MGDG), monogalactosylmonoacylglycerol (MGMG), digalactosyldiacylglycerol (DGDG) (Figure 2) plus digalactosylmonoacylglycerol (DGMG) classes, identified in the positive LC-MS spectra as $\left[\mathrm{M}+\mathrm{NH}_{4}\right]^{+}$ ions (Table 3). The most abundant species in each class of glycolipids was assigned as SQDG (36:5) corresponding to SQDG (20:5/16:0), SQMG (14:0), DGDG (36:5) as DGDG (20:5/16:0), DGMG(16:0), MGDG (40:10) as MGDG (20:5/20:5), and MGMG (20:5).

Table 2. Molecular species of glycolipids SQDGs and SQMGs identified by HILIC-ESI-MS as [M - H] ${ }^{-}$ ions. $\mathrm{C}$ represents the total number of carbon atoms and $\mathrm{N}$ represents the total number of double bonds on the fatty acyl chains. The most abundant species in each class are highlighted in bold type. Fatty acids' $s n-1$ and $s n-2$ position is based on biosynthetic pathways [27-29].

\begin{tabular}{cccccc}
\hline $\begin{array}{c}\text { Theoretical } \\
\boldsymbol{m} / \boldsymbol{z}\end{array}$ & $\begin{array}{c}\text { Observed } \\
\boldsymbol{m} / \boldsymbol{z}\end{array}$ & $\begin{array}{c}\text { Error } \\
(\mathbf{p p m})\end{array}$ & $\begin{array}{c}\text { Lipid Species } \\
\mathbf{( C : N )}\end{array}$ & Fatty Acyl Chains & Formula \\
\hline $\mathbf{5 2 7 . 2 5 2 6}$ & $\mathbf{5 2 7 . 2 5 4 1}$ & $\mathbf{2 . 8 4}$ & SQMG(14:0) & $\mathbf{1 4 : 0}$ & $\mathbf{C}_{23} \mathrm{H}_{43} \mathrm{O}_{11} \mathrm{~S}$ \\
555.2839 & 555.2855 & 2.88 & SQMG(16:0) & $16: 0$ & $\mathrm{C}_{25} \mathrm{H}_{47} \mathrm{O}_{11} \mathrm{~S}$ \\
737.4510 & 737.4529 & 2.58 & SQDG(28:0) & $14: 0 / 14: 0$ & $\mathrm{C}_{37} \mathrm{H}_{69} \mathrm{O}_{12} \mathrm{~S}$ \\
763.4666 & 763.4685 & 2.49 & SQDG(30:1) & $14: 0 / 16: 1$ & $\mathrm{C}_{39} \mathrm{H}_{71} \mathrm{O}_{12} \mathrm{~S}$ \\
765.4823 & 765.4797 & -3.40 & SQDG(30:0) & $14: 0 / 16: 0$ & $\mathrm{C}_{39} \mathrm{H}_{73} \mathrm{O}_{12} \mathrm{~S}$ \\
785.4510 & 785.4531 & 2.67 & SQDG(32:4) & $16: 3 / 16: 1$ & $\mathrm{C}_{41} \mathrm{H}_{69} \mathrm{O}_{12} \mathrm{~S}$ \\
787.4666 & 787.4630 & -4.57 & SQDG(32:3) & $16: 3 / 16: 0$ & $\mathrm{C}_{41} \mathrm{H}_{71} \mathrm{O}_{12} \mathrm{~S}$ \\
789.4823 & 789.4861 & 4.81 & SQDG(32:2) & $18: 2 / 14: 0$ & $\mathrm{C}_{41} \mathrm{H}_{73} \mathrm{O}_{12} \mathrm{~S}$ \\
791.4979 & 791.5001 & 2.78 & SQDG(32:1) & $18: 1 / 14: 0$ & $\mathrm{C}_{41} \mathrm{H}_{75} \mathrm{O}_{12} \mathrm{~S}$ \\
793.5136 & 793.5156 & 2.52 & SQDG(32:0) & $16: 0 / 16: 0$ & $\mathrm{C}_{41} \mathrm{H}_{77} \mathrm{O}_{12} \mathrm{~S}$ \\
811.4666 & 811.4685 & 2.34 & SQDG(34:5) & $20: 5 / 14: 0$ & $\mathrm{C}_{43} \mathrm{H}_{71} \mathrm{O}_{12} \mathrm{~S}$ \\
819.5292 & 819.5308 & 1.95 & SQDG(34:1) & $20: 1 / 14: 0$ & $\mathrm{C}_{43} \mathrm{H}_{79} \mathrm{O}_{12} \mathrm{~S}$ \\
821.5449 & 821.5461 & 1.46 & SQDG(34:0) & * & $\mathrm{C}_{43} \mathrm{H}_{81} \mathrm{O}_{12} \mathrm{~S}$ \\
837.4823 & 837.4834 & 1.31 & SQDG(36:6) & $20: 5 / 16: 1$ & $\mathrm{C}_{45} \mathrm{H}_{73} \mathrm{O}_{12} \mathrm{~S}$ \\
839.4979 & 839.4998 & $\mathbf{2 . 2 6}$ & SQDG(36:5) & $\mathbf{2 0 : 5 / 1 6 : 0}$ & $\mathrm{C}_{45} \mathrm{H}_{75} \mathrm{O}_{12} \mathrm{~S}$ \\
847.5605 & 847.5618 & 1.53 & SQDG(36:1) & $20: 1 / 16: 0$ & $\mathrm{C}_{45} \mathrm{H}_{83} \mathrm{O}_{12} \mathrm{~S}$ \\
859.4666 & 859.4682 & 1.86 & SQDG(38:9) & $22: 5 / 16: 4$ and 20:5/18:4 & $\mathrm{C}_{47} \mathrm{H}_{71} \mathrm{O}_{12} \mathrm{~S}$ \\
875.5918 & 875.5939 & 2.40 & SQDG(38:1) & $24: 1 / 14: 0$ & $\mathrm{C}_{47} \mathrm{H}_{87} \mathrm{O}_{12} \mathrm{~S}$ \\
885.4823 & 885.4843 & 2.26 & SQDG(40:10) & $20: 5 / 20: 5$ & $\mathrm{C}_{49} \mathrm{H}_{73} \mathrm{O}_{12} \mathrm{~S}$ \\
\hline
\end{tabular}

* No MS/MS information for FA composition. 


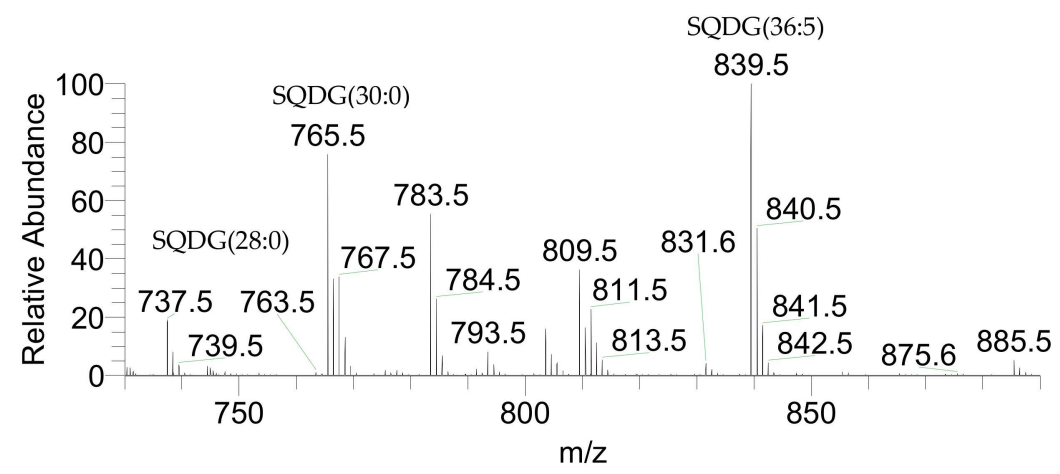

Figure 1. LC-MS spectrum representative of the class SQDG of P. palmata identified as $[\mathrm{M}-\mathrm{H}]^{-}$ions.

Betaine lipids of P. palmata included only one species of diacylglyceroltrimethylhomoserine (DGTS) assigned as DGTS (32:5), with a molecular formula of $\mathrm{C}_{42} \mathrm{H}_{80} \mathrm{O}_{7} \mathrm{~N}$, and identified in the LC-MS spectra as positive $[\mathrm{M}+\mathrm{H}]^{+}$ions. Its theoretical $\mathrm{m} / \mathrm{z}$ is 710.5935 and the observed $\mathrm{m} / \mathrm{z}$ was $710.5936(0.14 \mathrm{ppm})$.

Table 3. Molecular species of glycolipids MGDG, MGMG, DGDG, and DGMG identified by HILIC-ESI-MS as $[\mathrm{M}+\mathrm{NH} 4]^{+}$ions. $\mathrm{C}$ represents the total number of carbon atoms and $\mathrm{N}$ represents the total number of double bonds on the fatty acyl chains. The most abundant species in each class are highlighted in bold type. Fatty acids' $s n-1$ and $s n-2$ position is based on biosynthetic pathways [27-29].

\begin{tabular}{|c|c|c|c|c|c|}
\hline $\begin{array}{c}\text { Theoretical } \\
m / z\end{array}$ & $\begin{array}{c}\text { Observed } \\
m / z\end{array}$ & Error (ppm) & $\begin{array}{l}\text { Lipid Species } \\
\text { (C:N) }\end{array}$ & $\begin{array}{c}\text { Fatty Acyl } \\
\text { Chains }\end{array}$ & Formula \\
\hline 482.3329 & 482.3326 & -0.62 & MGMG(14:0) & $14: 0$ & $\mathrm{C}_{23} \mathrm{H}_{48} \mathrm{NO}_{9}$ \\
\hline 502.3016 & 502.3014 & -0.40 & MGMG(16:4) & $16: 4$ & $\mathrm{C}_{25} \mathrm{H}_{44} \mathrm{NO}_{9}$ \\
\hline 504.3173 & 504.3184 & 2.18 & MGMG(16:3) & $16: 3$ & $\mathrm{C}_{25} \mathrm{H}_{46} \mathrm{NO}_{9}$ \\
\hline 506.3329 & 506.3331 & 0.39 & MGMG(16:2) & $16: 2$ & $\mathrm{C}_{25} \mathrm{H}_{48} \mathrm{NO}_{9}$ \\
\hline 510.3642 & 510.3640 & -0.39 & MGMG(16:0) & $16: 0$ & $\mathrm{C}_{25} \mathrm{H}_{52} \mathrm{NO}_{9}$ \\
\hline 530.3329 & 530.3330 & 0.19 & MGMG(18:4) & $18: 4$ & $\mathrm{C}_{27} \mathrm{H}_{48} \mathrm{NO}_{9}$ \\
\hline 536.3799 & 536.3799 & 0.00 & MGMG(18:1) & $18: 1$ & $\mathrm{C}_{27} \mathrm{H}_{54} \mathrm{NO}_{9}$ \\
\hline 556.3486 & 556.3485 & -0.18 & MGMG(20:5) & $20: 5$ & $\mathrm{C}_{29} \mathrm{H}_{50} \mathrm{NO}_{9}$ \\
\hline 766.5469 & 766.5494 & 3.26 & MGDG(34:5) & $20: 5 / 14: 0$ & $\mathrm{C}_{43} \mathrm{H}_{76} \mathrm{NO}^{10}$ \\
\hline 774.6090 & 774.6104 & 1.81 & MGDG(34:1) & $18: 1 / 16: 0$ & $\mathrm{C}_{43} \mathrm{H}_{84} \mathrm{NO}_{10}$ \\
\hline 794.5782 & 794.5778 & -0.50 & MGDG(36:5) & $20: 5 / 16: 0$ & $\mathrm{C}_{45} \mathrm{H}_{80} \mathrm{NO}_{10}$ \\
\hline 822.6095 & 822.6101 & 0.73 & MGDG(38:5) & * & $\mathrm{C}_{47} \mathrm{H}_{84} \mathrm{NO}_{10}$ \\
\hline 840.5626 & 840.5627 & 0.12 & MGDG(40:10) & $20: 5 / 20: 5$ & $\mathrm{C}_{49} \mathrm{H}_{78} \mathrm{NO}_{10}$ \\
\hline 904.6878 & 904.6877 & -0.11 & MGDG(44:6) & * & $\mathrm{C}_{53} \mathrm{H}_{94} \mathrm{NO}_{10}$ \\
\hline 672.4170 & 672.4172 & 0.30 & DGMG(16:0) & $16: 0$ & $\mathrm{C}_{31} \mathrm{H}_{62} \mathrm{NO}_{14}$ \\
\hline 698.4327 & 698.4326 & -0.11 & DGMG(18:1) & $18: 1$ & $\mathrm{C}_{33} \mathrm{H}_{64} \mathrm{NO}_{14}$ \\
\hline 718.4014 & 718.4015 & 0.14 & DGMG(20:5) & $20: 5$ & $\mathrm{C}_{35} \mathrm{H}_{60} \mathrm{NO}_{14}$ \\
\hline 882.6154 & 882.6173 & 2.15 & DGDG(30:0) & * & $\mathrm{C}_{45} \mathrm{H}_{88} \mathrm{O}_{15} \mathrm{~N}$ \\
\hline 906.6154 & 906.6166 & 1.32 & DGDG(32:2) & * & $\mathrm{C}_{47} \mathrm{H}_{88} \mathrm{O}_{15} \mathrm{~N}$ \\
\hline 908.6310 & 908.6309 & -0.11 & DGDG(32:1) & 18:1/14:0 & $\mathrm{C}_{47} \mathrm{H}_{90} \mathrm{O}_{15} \mathrm{~N}$ \\
\hline 928.5997 & 928.5997 & 0.00 & DGDG(34:5) & $20: 5 / 14: 0$ & $\mathrm{C}_{49} \mathrm{H}_{86} \mathrm{O}_{15} \mathrm{~N}$ \\
\hline 932.6310 & 932.6319 & 0.97 & DGDG(34:3) & * & $\mathrm{C}_{49} \mathrm{H}_{90} \mathrm{O}_{15} \mathrm{~N}$ \\
\hline 934.6467 & 934.645 & -1.82 & DGDG(34:2) & * & $\mathrm{C}_{49} \mathrm{H}_{92} \mathrm{O}_{15} \mathrm{~N}$ \\
\hline 936.6623 & 936.6623 & 0.00 & DGDG(34:1) & 18:1/16:0 & $\mathrm{C}_{49} \mathrm{H}_{94} \mathrm{O}_{15} \mathrm{~N}$ \\
\hline 956.6310 & 956.6317 & 0.73 & DGDG(36:5) & $20: 5 / 16: 0$ & $\mathrm{C}_{51} \mathrm{H}_{90} \mathrm{O}_{15} \mathrm{~N}$ \\
\hline 984.6623 & 984.6617 & -0.61 & DGDG(38:5) & * & $\mathrm{C}_{53} \mathrm{H}_{94} \mathrm{O}_{15} \mathrm{~N}$ \\
\hline 1002.6154 & 1002.6160 & 0.60 & DGDG(40:10) & $20: 5 / 20: 5$ & $\mathrm{C}_{55} \mathrm{H}_{88} \mathrm{O}_{15} \mathrm{~N}$ \\
\hline
\end{tabular}

${ }^{*}$ No MS/MS information for FA composition.

The classes of phospholipids found in P. palmata included phosphatidylcholine (PC) (Figure 3A), lyso-PC (LPC), phosphatilylethanolanime (PE) (Figure 3B), and lyso-PE (LPE) were identified in the LC-MS spectra as positive $[\mathrm{M}+\mathrm{H}]^{+}$ions (Table 4). Phosphatidylglycerol (PG) (Figure 4), lyso-PG (LPG), phosphatidylinositol (PI), and phosphatidic acid (PA) were identified in the LC-MS spectra as negative 
$[\mathrm{M}-\mathrm{H}]^{-}$ions (Table 5). Also, inositephosphoceramide lipids (IPC) were identified as negative $[\mathrm{M}+$ $\mathrm{CH}_{3} \mathrm{COO}^{-}$ions (Table 6). The PC(40:10) identified as PC(20:5/20:5), LPC(20:5), PE(40:10) identified as PE(20:5/20:5), LPE(20:5), PG(36:6) as PG(16:1/20:5), LPG(14:0), PI(40:10) as PI(20:5/20:5), PA(40:10) as PA(20:5/20:5), and PI-Cer(d40:2) represent the most abundant species within each phospholipid class.

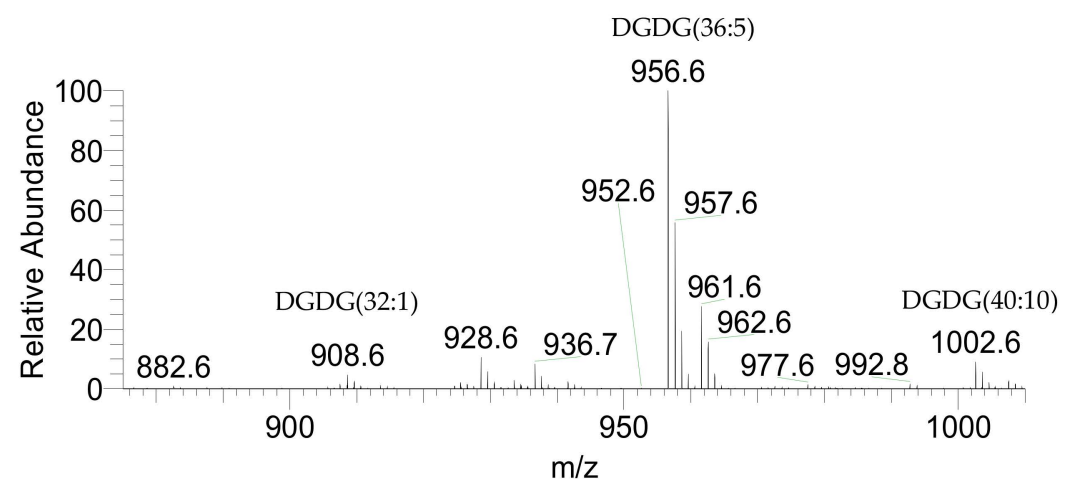

Figure 2. LC-MS spectrum representative of P. palmata DGDG class identified as $\left[\mathrm{M}+\mathrm{NH}_{4}\right]^{+}$ions.

Table 4. Molecular species of phospholipids LPC, PC, LPE, and PE identified by HILIC-ESI-MS as positive $[\mathrm{M}+\mathrm{H}]^{+}$ions. $\mathrm{C}$ represents the total number of carbon atoms and $\mathrm{N}$ represents the total number of double bonds on the fatty acyl chains. The most abundant species in each class are highlighted in bold type. Fatty acids' $s n-1$ and $s n-2$ position is based on biosynthetic pathways [27-29].

\begin{tabular}{|c|c|c|c|c|c|}
\hline $\begin{array}{c}\text { Theoretical } \\
m / z\end{array}$ & $\begin{array}{c}\text { Observed } \\
m / z\end{array}$ & $\begin{array}{l}\text { Error } \\
(\mathrm{ppm})\end{array}$ & $\begin{array}{c}\text { Lipid Species } \\
\text { (C:N) }\end{array}$ & Fatty Acyl Chains & Formula \\
\hline 468.3090 & 468.3092 & 0.43 & $\operatorname{LPC}(14: 0)$ & $14: 0$ & $\mathrm{C}_{22} \mathrm{H}_{47} \mathrm{NO}_{7} \mathrm{P}$ \\
\hline 494.3247 & 494.3242 & -1.01 & $\operatorname{LPC}(16: 1)$ & $16: 1$ & $\mathrm{C}_{24} \mathrm{H}_{49} \mathrm{NO}_{7} \mathrm{P}$ \\
\hline 496.3403 & 496.3401 & -0.40 & $\operatorname{LPC}(16: 0)$ & $16: 0$ & $\mathrm{C}_{24} \mathrm{H}_{51} \mathrm{NO}_{7} \mathrm{P}$ \\
\hline 516.3090 & 516.3090 & 0.00 & LPC(18:4) & $18: 4$ & $\mathrm{C}_{26} \mathrm{H}_{47} \mathrm{NO}_{7} \mathrm{P}$ \\
\hline 518.3247 & 518.3236 & -2.12 & $\operatorname{LPC}(18: 3)$ & $18: 3$ & $\mathrm{C}_{26} \mathrm{H}_{49} \mathrm{NO}_{7} \mathrm{P}$ \\
\hline 520.3403 & 520.3395 & -1.54 & $\operatorname{LPC}(18: 2)$ & $18: 2$ & $\mathrm{C}_{26} \mathrm{H}_{51} \mathrm{NO}_{7} \mathrm{P}$ \\
\hline 522.3560 & 522.3564 & 0.77 & $\operatorname{LPC}(18: 1)$ & $18: 1$ & $\mathrm{C}_{26} \mathrm{H}_{53} \mathrm{NO}_{7} \mathrm{P}$ \\
\hline 542.3247 & 542.3246 & -0.18 & $\operatorname{LPC}(20: 5)$ & $20: 5$ & $\mathrm{C}_{28} \mathrm{H}_{49} \mathrm{NO}_{7} \mathrm{P}$ \\
\hline 568.3403 & 568.3405 & 0.35 & $\operatorname{LPC}(22: 6)$ & $22: 6$ & $\mathrm{C}_{30} \mathrm{H}_{51} \mathrm{NO}_{7} \mathrm{P}$ \\
\hline 570.3560 & 570.3559 & -0.18 & $\operatorname{LPC}(22: 5)$ & $22: 5$ & $\mathrm{C}_{30} \mathrm{H}_{53} \mathrm{NO}_{7} \mathrm{P}$ \\
\hline 706.5387 & 706.5387 & 0.00 & PC $(30: 0)$ & $14: 0 / 16: 0$ & $\mathrm{C}_{38} \mathrm{H}_{77} \mathrm{NO}_{8} \mathrm{P}$ \\
\hline 724.4917 & 724.4916 & -0.14 & $\mathrm{PC}(32: 5)$ & * & $\mathrm{C}_{40} \mathrm{H}_{71} \mathrm{NO}_{8} \mathrm{P}$ \\
\hline 726.5074 & 726.5070 & -0.55 & PC $(32: 4)$ & $14: 0 / 18: 4$ & $\mathrm{C}_{40} \mathrm{H}_{73} \mathrm{NO}_{8} \mathrm{P}$ \\
\hline 728.5230 & 728.5233 & 0.41 & $\operatorname{PC}(32: 3)$ & $14: 0 / 18: 3$ & $\mathrm{C}_{40} \mathrm{H}_{75} \mathrm{NO}_{8} \mathrm{P}$ \\
\hline 730.5387 & 730.5387 & 0.00 & PC (32:2) & $14: 0 / 18: 2$ and $16: 0 / 16: 2$ & $\mathrm{C}_{40} \mathrm{H}_{77} \mathrm{NO}_{8} \mathrm{P}$ \\
\hline 732.5543 & 732.5541 & -0.27 & PC $(32: 1)$ & $16: 0 / 16: 1$ and $14: 0 / 18: 1$ & $\mathrm{C}_{40} \mathrm{H}_{79} \mathrm{NO}_{8} \mathrm{P}$ \\
\hline 734.5700 & 734.5696 & -0.54 & $\mathrm{PC}(32: 0)$ & $*$ & $\mathrm{C}_{40} \mathrm{H}_{81} \mathrm{NO}_{8} \mathrm{P}$ \\
\hline 750.5074 & 750.5059 & -2.00 & PC $(34: 6)$ & * & $\mathrm{C}_{42} \mathrm{H}_{73} \mathrm{NO}_{8} \mathrm{P}$ \\
\hline 752.5230 & 752.523 & 0.00 & PC(34:5) & $14: 0 / 20: 5$ & $\mathrm{C}_{42} \mathrm{H}_{75} \mathrm{NO}_{8} \mathrm{P}$ \\
\hline 754.5387 & 754.538 & -0.93 & PC $(34: 4)$ & $16: 0 / 18: 4$ & $\mathrm{C}_{42} \mathrm{H}_{77} \mathrm{NO}_{8} \mathrm{P}$ \\
\hline 756.5543 & 756.5541 & -0.26 & PC (34:3) & $16: 0 / 18: 3$ & $\mathrm{C}_{42} \mathrm{H}_{79} \mathrm{NO}_{8} \mathrm{P}$ \\
\hline 758.5700 & 758.5699 & -0.13 & PC $(34: 2)$ & $16: 0 / 18: 2$ & $\mathrm{C}_{42} \mathrm{H}_{81} \mathrm{NO}_{8} \mathrm{P}$ \\
\hline 760.5856 & 760.5856 & 0.00 & PC $(34: 1)$ & $16: 0 / 18: 1$ & $\mathrm{C}_{42} \mathrm{H}_{83} \mathrm{NO}_{8} \mathrm{P}$ \\
\hline 774.5073 & 774.5058 & -1.94 & $\mathrm{PC}(36: 8)$ & $16: 3 / 20: 5$ & $\mathrm{C}_{44} \mathrm{H}_{73} \mathrm{NO}_{8} \mathrm{P}$ \\
\hline 776.5230 & 776.5215 & -1.93 & PC $(36: 7)$ & $16: 2 / 20: 5$ & $\mathrm{C}_{44} \mathrm{H}_{75} \mathrm{NO}_{8} \mathrm{P}$ \\
\hline 778.5387 & 778.5386 & -0.13 & $\mathrm{PC}(36: 6)$ & $16: 1 / 20: 5$ & $\mathrm{C}_{44} \mathrm{H}_{77} \mathrm{NO}_{8} \mathrm{P}$ \\
\hline 780.5543 & 780.5544 & 0.13 & PC $(36: 5)$ & $16: 0 / 20: 5$ & $\mathrm{C}_{44} \mathrm{H}_{79} \mathrm{NO}_{8} \mathrm{P}$ \\
\hline 784.5856 & 784.5823 & -4.21 & PC $(36: 3)$ & $*$ & $\mathrm{C}_{44} \mathrm{H}_{83} \mathrm{NO}_{8} \mathrm{P}$ \\
\hline 786.6013 & 786.6002 & -1.40 & PC $(36: 2)$ & * & $\mathrm{C}_{44} \mathrm{H}_{85} \mathrm{NO}_{8} \mathrm{P}$ \\
\hline 788.6169 & 788.6153 & -2.03 & PC $(36: 1)$ & $14: 0 / 22: 1$ & $\mathrm{C}_{44} \mathrm{H}_{87} \mathrm{NO}_{8} \mathrm{P}$ \\
\hline 800.5230 & 800.5225 & -0.62 & PC (38:9) & $18: 4 / 20: 5$ & $\mathrm{C}_{46} \mathrm{H}_{75} \mathrm{NO}_{8} \mathrm{P}$ \\
\hline 802.5387 & 802.5362 & -3.12 & PC $(38: 8)$ & $18: 3 / 20: 5$ & $\mathrm{C}_{46} \mathrm{H}_{77} \mathrm{NO}_{8} \mathrm{P}$ \\
\hline
\end{tabular}


Table 4. Cont.

\begin{tabular}{|c|c|c|c|c|c|}
\hline $\begin{array}{c}\text { Theoretical } \\
m / z\end{array}$ & $\begin{array}{c}\text { Observed } \\
m / z\end{array}$ & $\begin{array}{l}\text { Error } \\
(\mathrm{ppm})\end{array}$ & $\begin{array}{l}\text { Lipid Species } \\
\text { (C:N) }\end{array}$ & Fatty Acyl Chains & Formula \\
\hline 806.5700 & 806.5699 & -0.12 & $\mathrm{PC}(38: 6)$ & $18: 1 / 20: 5$ & $\mathrm{C}_{46} \mathrm{H}_{81} \mathrm{NO}_{8} \mathrm{P}$ \\
\hline 808.5856 & 808.5845 & -1.36 & PC (38:5) & $18: 0 / 20: 5$ & $\mathrm{C}_{46} \mathrm{H}_{83} \mathrm{NO}_{8} \mathrm{P}$ \\
\hline 810.6013 & 810.597 & -5.30 & PC $(38: 4)$ & * & $\mathrm{C}_{46} \mathrm{H}_{85} \mathrm{NO}_{8} \mathrm{P}$ \\
\hline 816.6482 & 816.6486 & 0.49 & PC $(38: 1)$ & * & $\mathrm{C}_{46} \mathrm{H}_{91} \mathrm{NO}_{8} \mathrm{P}$ \\
\hline 826.5387 & 826.5391 & 0.48 & PC(40:10) & $20: 5 / 20: 5$ & $\mathrm{C}_{48} \mathrm{H}_{77} \mathrm{NO}_{8} \mathrm{P}$ \\
\hline 834.6013 & 834.5997 & -1.92 & PC(40:6) & $20: 1 / 20: 5$ & $\mathrm{C}_{48} \mathrm{H}_{85} \mathrm{NO}_{8} \mathrm{P}$ \\
\hline 836.6169 & 836.6153 & -1.91 & $\mathrm{PC}(40: 5)$ & * & $\mathrm{C}_{48} \mathrm{H}_{87} \mathrm{NO}_{8} \mathrm{P}$ \\
\hline 854.5700 & 854.5696 & -0.47 & PC $(42: 10)$ & $22: 5 / 20: 5$ & $\mathrm{C}_{50} \mathrm{H}_{81} \mathrm{NO}_{8} \mathrm{P}$ \\
\hline 862.6326 & 862.6334 & 0.93 & PC $(42: 6)$ & $22: 1 / 20: 5$ & $\mathrm{C}_{50} \mathrm{H}_{89} \mathrm{NO}_{8} \mathrm{P}$ \\
\hline 864.6482 & 864.6459 & -2.66 & PC(42:5) & $22: 0 / 20: 5$ & $\mathrm{C}_{50} \mathrm{H}_{91} \mathrm{NO}_{8} \mathrm{P}$ \\
\hline 882.6013 & 882.6026 & 1.47 & PC $(44: 10)$ & * & $\mathrm{C}_{52} \mathrm{H}_{85} \mathrm{NO}_{8} \mathrm{P}$ \\
\hline 890.6639 & 890.6639 & 0.00 & $\mathrm{PC}(44: 6)$ & $24: 1 / 20: 5$ & $\mathrm{C}_{52} \mathrm{H}_{93} \mathrm{NO}_{8} \mathrm{P}$ \\
\hline 480.3090 & 480.3092 & 0.42 & LPE(18:1) & $18: 1$ & $\mathrm{C}_{23} \mathrm{H}_{47} \mathrm{NO}_{7} \mathrm{P}$ \\
\hline 500.2777 & 500.2776 & -0.20 & LPE(20:5) & $20: 5$ & $\mathrm{C}_{25} \mathrm{H}_{43} \mathrm{NO}_{7} \mathrm{P}$ \\
\hline 658.4448 & 658.4426 & -3.34 & $\operatorname{PE}(30: 3)$ & * & $\mathrm{C}_{35} \mathrm{H}_{65} \mathrm{NO}_{8} \mathrm{P}$ \\
\hline 688.4917 & 688.4936 & 2.76 & $\operatorname{PE}(32: 2)$ & $16: 1 / 16: 1$ & $\mathrm{C}_{37} \mathrm{H}_{71} \mathrm{NO}_{8} \mathrm{P}$ \\
\hline 690.5074 & 690.5088 & 2.03 & $\operatorname{PE}(32: 1)$ & $16: 0 / 16: 1$ and $14: 0 / 18: 1$ & $\mathrm{C}_{37} \mathrm{H}_{73} \mathrm{NO}_{8} \mathrm{P}$ \\
\hline 710.4761 & 710.4753 & -1.13 & $\operatorname{PE}(34: 5)$ & $14: 0 / 20: 5$ & $\mathrm{C}_{39} \mathrm{H}_{69} \mathrm{NO}_{8} \mathrm{P}$ \\
\hline 712.4917 & 712.4887 & -4.21 & $\operatorname{PE}(34: 4)$ & $16: 0 / 18: 4$ & $\mathrm{C}_{39} \mathrm{H}_{71} \mathrm{NO}_{8} \mathrm{P}$ \\
\hline 714.5074 & 714.5084 & 1.40 & $\operatorname{PE}(34: 3)$ & $16: 0 / 18: 3$ and $16: 1 / 18: 2$ & $\mathrm{C}_{39} \mathrm{H}_{73} \mathrm{NO}_{8} \mathrm{P}$ \\
\hline 716.5230 & 716.5233 & 0.42 & $\operatorname{PE}(34: 2)$ & $16: 1 / 18: 1$ and $16: 0 / 18: 2$ & $\mathrm{C}_{39} \mathrm{H}_{75} \mathrm{NO}_{8} \mathrm{P}$ \\
\hline 718.5387 & 718.5385 & -0.28 & $\operatorname{PE}(34: 1)$ & $16: 0 / 18: 1$ & $\mathrm{C}_{39} \mathrm{H}_{77} \mathrm{NO}_{8} \mathrm{P}$ \\
\hline 736.4917 & 736.4912 & -0.68 & $\operatorname{PE}(36: 6)$ & * & $\mathrm{C}_{41} \mathrm{H}_{71} \mathrm{NO}_{8} \mathrm{P}$ \\
\hline 738.5074 & 738.5073 & -0.14 & $\operatorname{PE}(36: 5)$ & $16: 0 / 20: 5$ & $\mathrm{C}_{41} \mathrm{H}^{73} \mathrm{NO}_{8} \mathrm{P}$ \\
\hline 742.5387 & 742.5381 & -0.81 & $\operatorname{PE}(36: 3)$ & * & $\mathrm{C}_{41} \mathrm{H}_{77} \mathrm{NO}_{8} \mathrm{P}$ \\
\hline 760.4917 & 760.489 & -3.55 & $\operatorname{PE}(38: 8)$ & * & $\mathrm{C}_{43} \mathrm{H}_{71} \mathrm{NO}_{8} \mathrm{P}$ \\
\hline 764.5230 & 764.5239 & 1.18 & $\operatorname{PE}(38: 6)$ & $18: 1 / 20: 5$ & $\mathrm{C}_{43} \mathrm{H}_{75} \mathrm{NO}_{8} \mathrm{P}$ \\
\hline 766.5387 & 766.5396 & 1.17 & $\operatorname{PE}(38: 5)$ & $16: 0 / 22: 5$ and $18: 0 / 20: 5$ & $\mathrm{C}_{43} \mathrm{H}_{77} \mathrm{NO}_{8} \mathrm{P}$ \\
\hline 784.4917 & 784.492 & 0.38 & PE(40:10) & $20: 5 / 20: 5$ & $\mathrm{C}_{45} \mathrm{H}_{71} \mathrm{NO}_{8} \mathrm{P}$ \\
\hline
\end{tabular}
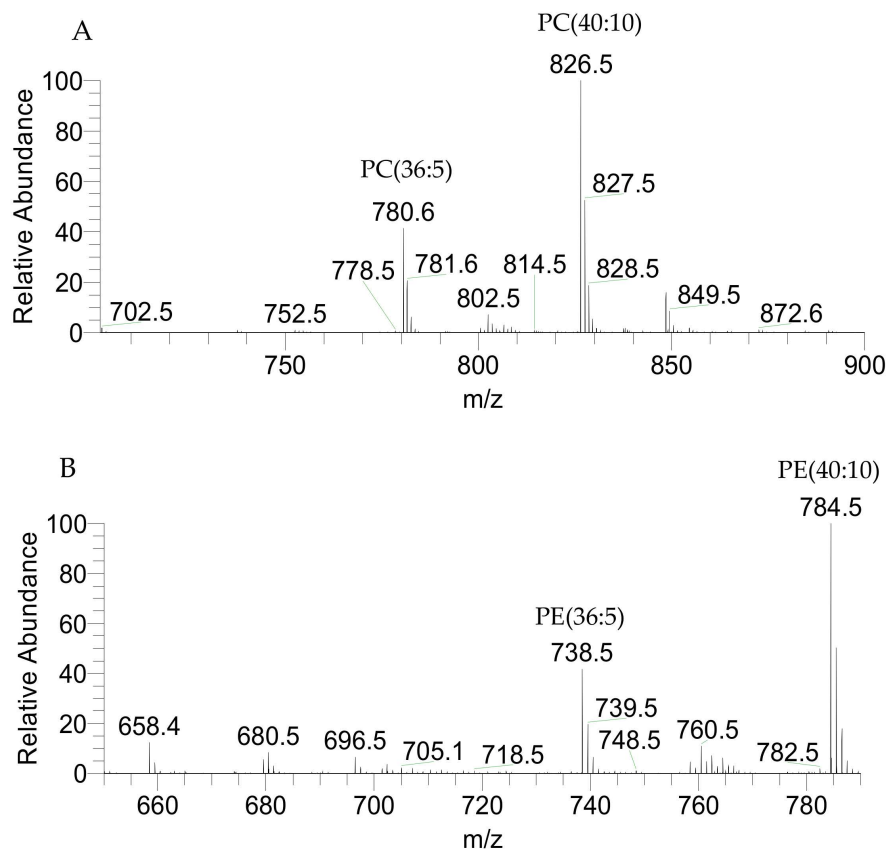

Figure 3. LC-MS representative spectra of P. palmata PC (A) and PE (B) classes identified as $[\mathrm{M}+\mathrm{H}]^{+}$ions. 
Table 5. Molecular species of phospholipids LPG, PG, PI, and PA identified by HILIC-ESI-MS as negative $[\mathrm{M}-\mathrm{H}]^{-}$ions. $\mathrm{C}$ represents the total number of carbon atoms and $\mathrm{N}$ represents the total number of double bonds on the fatty acyl chains. The most abundant species in each class are highlighted in bold type. Fatty acids' $s n-1$ and $s n-2$ position is based on biosynthetic pathways [27-29].

\begin{tabular}{|c|c|c|c|c|c|}
\hline $\begin{array}{c}\text { Theoretical } \\
m / z\end{array}$ & $\begin{array}{c}\text { Observed } \\
\mathrm{m} / \mathrm{z}\end{array}$ & $\begin{array}{l}\text { Error } \\
(\mathrm{ppm})\end{array}$ & $\begin{array}{l}\text { Lipid Species } \\
\text { (C:N) }\end{array}$ & Fatty Acyl Chains & Formula \\
\hline 455.2410 & 455.2425 & 3.29 & LPG(14:0) & 14:0 & $\mathrm{C}_{20} \mathrm{H}_{40} \mathrm{O}_{9} \mathrm{P}$ \\
\hline 481.2566 & 481.258 & 2.91 & LPG(16:1) & $16: 1$ & $\mathrm{C}_{22} \mathrm{H}_{42} \mathrm{O}_{9} \mathrm{P}$ \\
\hline 483.2723 & 483.2741 & 3.72 & LPG(16:0) & $16: 0$ & $\mathrm{C}_{22} \mathrm{H}_{44} \mathrm{O}_{9} \mathrm{P}$ \\
\hline 529.2566 & 529.2583 & 3.21 & LPG(20:5) & $20: 5$ & $\mathrm{C}_{26} \mathrm{H}_{42} \mathrm{O}_{9} \mathrm{P}$ \\
\hline 691.4550 & 691.4565 & 2.17 & PG(30:1) & $14: 0 / 16: 1$ & $\mathrm{C}_{36} \mathrm{H}_{68} \mathrm{O}_{10} \mathrm{P}$ \\
\hline 693.4707 & 693.4724 & 2.45 & PG(30:0) & $14: 0 / 16: 0$ & $\mathrm{C}_{36} \mathrm{H}_{70} \mathrm{O}_{10} \mathrm{P}$ \\
\hline 719.4863 & 719.4879 & 2.22 & PG(32:1) & $14: 0 / 18: 1$ and $16: 0 / 16: 1$ & $\mathrm{C}_{38} \mathrm{H}_{72} \mathrm{O}_{10} \mathrm{P}$ \\
\hline 721.502 & 721.5039 & 2.63 & PG(32:0) & $16: 0 / 16: 0$ and $14: 0 / 18: 0$ & $\mathrm{C}_{38} \mathrm{H}_{74} \mathrm{O}_{10} \mathrm{P}$ \\
\hline 739.455 & 739.4583 & 4.46 & PG(34:5) & $14: 0 / 20: 5$ & $\mathrm{C}_{40} \mathrm{H}_{68} \mathrm{O}_{10} \mathrm{P}$ \\
\hline 745.502 & 745.5033 & 1.74 & PG(34:2) & $16: 0 / 18: 2$ and $16: 1 / 18: 1$ & $\mathrm{C}^{40} \mathrm{H}_{74} \mathrm{O}_{10} \mathrm{P}$ \\
\hline 747.5176 & 747.5189 & 1.74 & PG(34:1) & $16: 0 / 18: 1$ and $14: 0 / 20: 1$ & $\mathrm{C}_{40} \mathrm{H}_{76} \mathrm{O}_{10} \mathrm{P}$ \\
\hline 749.5333 & 749.5345 & 1.60 & PG(34:0) & $16: 0 / 18: 0$ and $14: 0 / 20: 0$ & $\mathrm{C}_{40} \mathrm{H}_{78} \mathrm{O}_{10} \mathrm{P}$ \\
\hline 765.4707 & 765.4735 & 3.66 & PG(36:6) & $16: 1 / 20: 5$ & $\mathrm{C}_{40} \mathrm{H}_{70} \mathrm{O}_{10} \mathrm{P}$ \\
\hline 767.4863 & 767.4871 & 1.04 & PG(36:5) & * & $\mathrm{C}_{42} \mathrm{H}_{72} \mathrm{O}_{10} \mathrm{P}$ \\
\hline 773.5333 & 773.5348 & 1.94 & PG(36:2) & $18: 1 / 18: 1$ & $\mathrm{C}_{42} \mathrm{H}_{78} \mathrm{O}_{10} \mathrm{P}$ \\
\hline 775.5489 & 775.5508 & 2.45 & PG(36:1) & $14: 0 / 22: 1$ & $\mathrm{C}_{42} \mathrm{H}_{80} \mathrm{O}_{10} \mathrm{P}$ \\
\hline 777.5646 & 777.5662 & 2.06 & PG(36:0) & * & $\mathrm{C}_{42} \mathrm{H}_{82} \mathrm{O}_{10} \mathrm{P}$ \\
\hline 783.4812 & 783.4810 & -0.29 & PG(36:5-OH) & $16: 0-\mathrm{OH} / 20: 5$ & $\mathrm{C}_{42} \mathrm{H}_{72} \mathrm{O}_{11} \mathrm{P}$ \\
\hline 801.5646 & 801.5668 & 2.74 & PG(38:2) & $16: 0 / 24: 2$ & $\mathrm{C}_{44} \mathrm{H}_{82} \mathrm{O}_{10} \mathrm{P}$ \\
\hline 805.5959 & 805.5945 & -1.74 & PG(38:0) & * & $\mathrm{C}_{44} \mathrm{H}_{86} \mathrm{O}_{10} \mathrm{P}$ \\
\hline 813.4707 & 813.4736 & 3.60 & PG(40:10) & $20: 5 / 20: 5$ & $\mathrm{C}_{46} \mathrm{H}_{70} \mathrm{O}_{10} \mathrm{P}$ \\
\hline 829.5959 & 829.599 & 3.74 & PG(40:2) & $16: 1 / 24: 1$ and $14: 0 / 26: 2$ & $\mathrm{C}_{46} \mathrm{H}_{86} \mathrm{O}_{10} \mathrm{P}$ \\
\hline 831.6115 & 831.6138 & 2.77 & PG(40:1) & $14: 0 / 26: 1$ & $\mathrm{C}_{46} \mathrm{H}_{88} \mathrm{O}_{10} \mathrm{P}$ \\
\hline 859.6428 & 859.6452 & 2.79 & PG(42:1) & $16: 0 / 26: 1$ & $\mathrm{C}_{48} \mathrm{H}_{92} \mathrm{O}_{10} \mathrm{P}$ \\
\hline 855.5024 & 855.5039 & 1.75 & PI(36:5) & $16: 0 / 20: 5$ & $\mathrm{C}_{45} \mathrm{H}_{76} \mathrm{O}_{13} \mathrm{P}$ \\
\hline 881.5180 & 881.5204 & 2.72 & $\mathrm{PI}(38: 6)$ & $18: 1 / 20: 5$ & $\mathrm{C}_{47} \mathrm{H}_{78} \mathrm{O}_{13} \mathrm{P}$ \\
\hline 901.4867 & 901.4888 & 2.33 & PI(40:10) & $20: 5 / 20: 5$ & $\mathrm{C}_{49} \mathrm{H}_{74} \mathrm{O}_{13} \mathrm{P}$ \\
\hline 965.6119 & 965.6138 & 1.97 & PI(44:6) & $*$ & $\mathrm{C}_{53} \mathrm{H}_{90} \mathrm{O}_{13} \mathrm{P}$ \\
\hline 739.4339 & 739.4346 & 0.95 & $\operatorname{PA}(40: 10)$ & $20: 5 / 20: 5$ & $\mathrm{C}_{43} \mathrm{H}_{64} \mathrm{O}_{8} \mathrm{P}$ \\
\hline 743.4652 & 743.4669 & 2.29 & $\mathrm{PA}(40: 8)$ & $20: 4 / 20: 4$ & $\mathrm{C}_{43} \mathrm{H}_{68} \mathrm{O}_{8} \mathrm{P}$ \\
\hline
\end{tabular}

* No MS/MS information for FA composition.

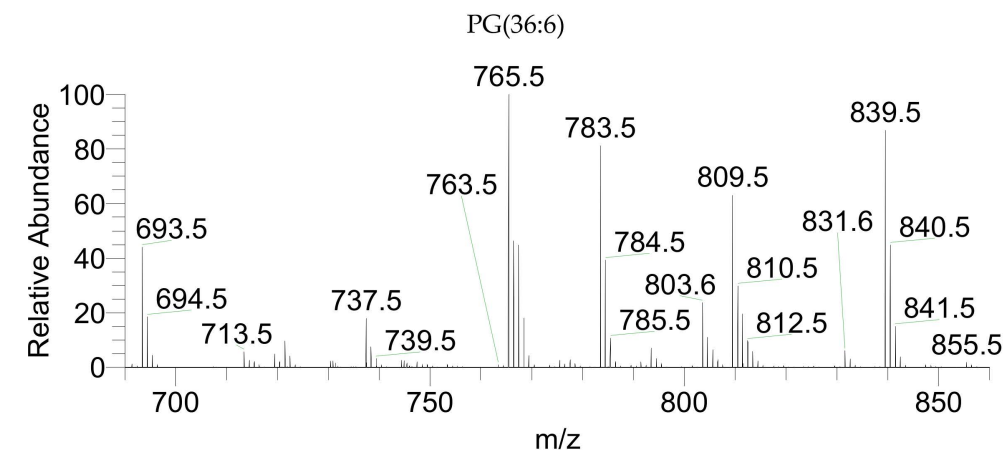

Figure 4. LC-MS representative spectrum of P. palmata PG class identified as $[\mathrm{M}-\mathrm{H}]^{-}$ions. 
Table 6. Molecular species of IPC identified by HILIC-ESI-MS as negative $\left[\mathrm{M}+\mathrm{CH}_{3} \mathrm{COO}\right]^{-}$ions. C represents the total number of carbon atoms and $\mathrm{N}$ represents the total number of double bonds on the fatty acyl chains. The most abundant species are highlighted in bold type.

\begin{tabular}{cccccc}
\hline $\begin{array}{c}\text { Theoretical } \\
\mathbf{m} / \boldsymbol{z}\end{array}$ & $\begin{array}{c}\text { Observed } \\
\mathbf{m} \boldsymbol{z}\end{array}$ & Error (ppm) & $\begin{array}{c}\text { Lipid Species } \\
(\mathrm{C}: \mathrm{N})\end{array}$ & $\begin{array}{c}\text { Fatty Acyl } \\
\text { Chains }\end{array}$ & Formula \\
\hline 892.5915 & 892.5933 & 2.02 & PI-Cer(d38:2) & $*$ & $\mathrm{C}_{46} \mathrm{H}_{87} \mathrm{NO}_{13} \mathrm{P}$ \\
918.6072 & 918.6096 & 2.61 & PI-Cer(d40:3) & $*$ & $\mathrm{C}_{48} \mathrm{H}_{89} \mathrm{NO}_{13} \mathrm{P}$ \\
$\mathbf{9 2 0 . 6 2 2 8}$ & $\mathbf{9 2 0 . 6 2 1 7}$ & $\mathbf{- 1 . 1 9}$ & PI-Cer(d40:2) & $*$ & $\mathrm{C}_{48} \mathrm{H}_{91} \mathrm{NO}_{13} \mathrm{P}$ \\
922.6385 & 922.6353 & -3.47 & PI-Cer(d40:1) & $*$ & $\mathrm{C}_{48} \mathrm{H}_{93} \mathrm{NO}_{13} \mathrm{P}$ \\
946.6385 & 946.6403 & 1.90 & PI-Cer(d42:3) & $*$ & $\mathrm{C}_{50} \mathrm{H}_{93} \mathrm{NO}_{13} \mathrm{P}$ \\
948.6541 & 948.6535 & -0.63 & PI-Cer(d42:2) & $*$ & $\mathrm{C}_{50} \mathrm{H}_{95} \mathrm{NO}_{13} \mathrm{P}$ \\
\hline
\end{tabular}

* No MS/MS information for FA composition.

The relative quantification of the identified species was calculated after integrating the peak area of each identified ion, normalized to the internal standard and divided by the sum of the normalized area of all species. Lipid species identified with a relative percentage of $0.5 \%$ or greater are shown in Figure 5 . Phospholipids were the group of lipids with the largest number of species (91 species). Particular emphasis is placed on PC(40:10), assigned as PC(20:5/20:5), which was the most abundant identified lipid species, with an average relative percentage of $23.7 \%$. The PC(36:5), assigned as PC(16:0/20:5) was the second most abundant species, with an average relative percentage of $10.4 \%$. It remains important to highlight the lipid species SQDG(36:5), MGDG(40:10), DGDG(36:5), SQDG(30:0), and MGMG(20:5), with an average relative percentage of $5 \%$ or greater. Both PC species are EPA carriers. The 20:5 fatty acyl chain was confirmed by LC-MS/MS spectra data analysis of the above-mentioned species, with the exception of SQDG (30:0). Indeed, the lipid molecular species with 5 and 10 double bonds on the fatty acid acyl chains are clearly pronounced (Figure 6), corroborating the prevalence of EPA.

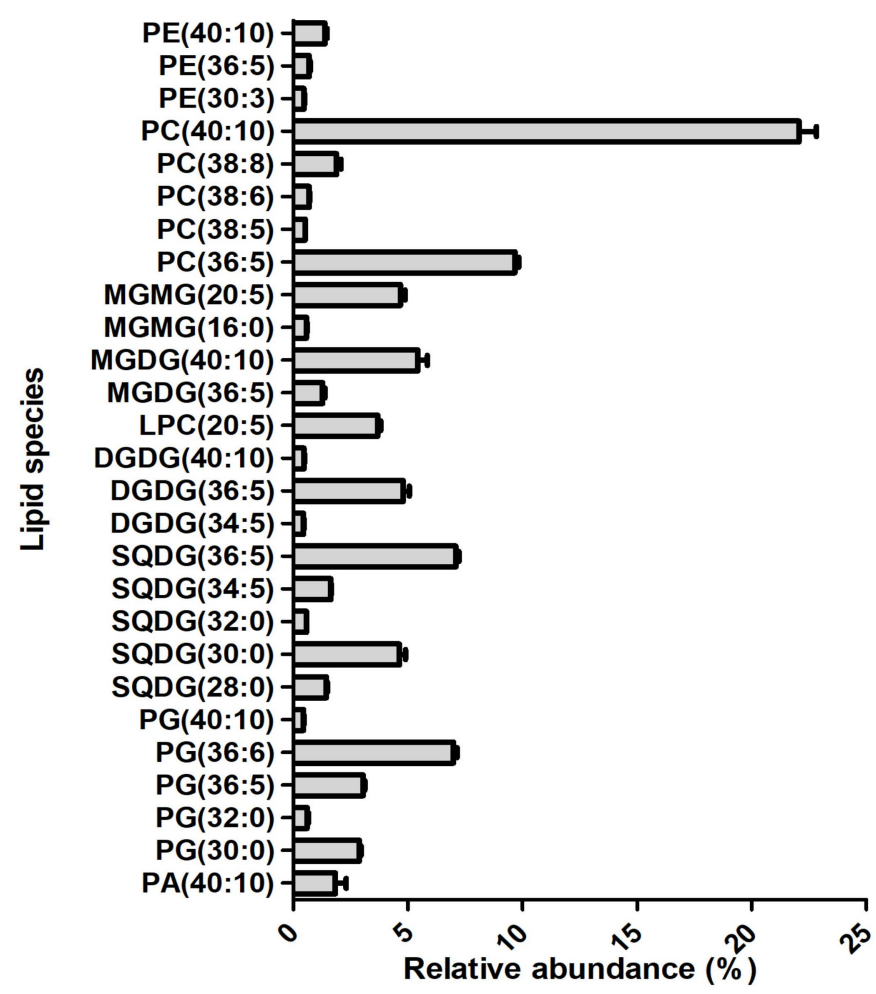

Figure 5. The relative percentage of lipid contents equal to or greater than $0.5 \%$ of P. palmata. Values are averages of five samples \pm standard deviation. 


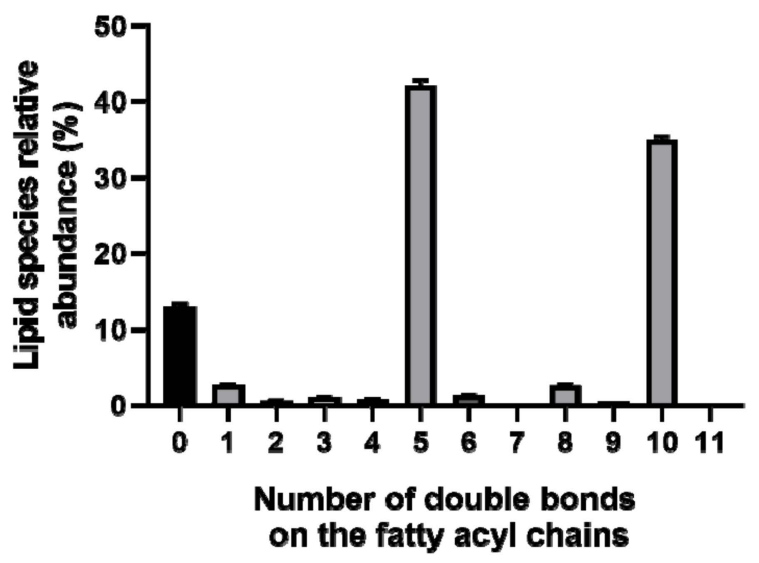

Figure 6. The relative abundance of lipid species of $P$. palmata including the same number of double bonds on fatty acyl chains.

\subsection{Evaluation of Antioxidant Activity}

The antioxidant potential of P. palmata polar lipid-rich extract was evaluated through free radical $\mathrm{DPPH}^{\bullet}$ and $\mathrm{ABTS}^{\bullet+}$ scavenging assays. While DPPH assay measures the ability of antioxidants to scavenge the $\mathrm{DPPH}^{\bullet}$ generated in the organic phase, the $\mathrm{ABTS}^{\bullet+}$ assay acts in the same way in the aqueous phase. The percentage of radical inhibition in the presence of extracts rich in polar lipids was calculated after $120 \mathrm{~min}$. The percent inhibition is proportional to time and the lipid extract concentration, even though the response appeared to be non-linear, with better performance for the $\mathrm{ABTS}^{\bullet+}$ assay (Figure 7).
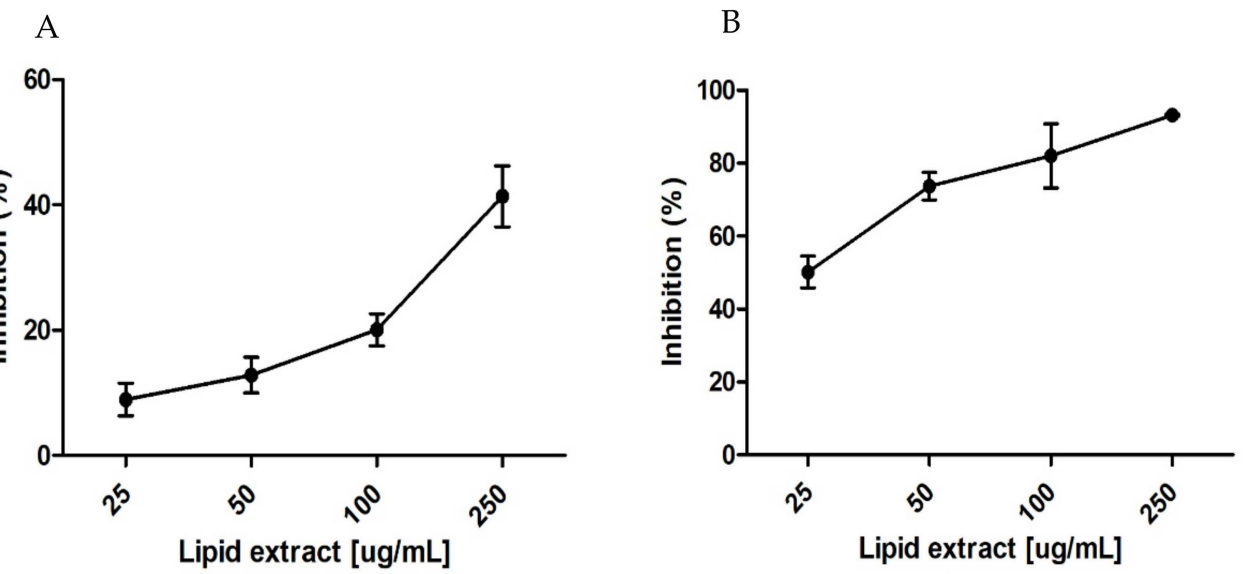

Figure 7. Percent inhibition as a function of the concentration of the lipid extract after $120 \mathrm{~min}$ of $\mathrm{DPPH}^{\bullet}(\mathbf{A})$ and $\mathrm{ABTS}^{\bullet+}(\mathbf{B})$ radical scavenging activity.

Results gathered in these assays showed that for the DPPH assay, the polar lipid-rich extract concentration providing $30 \%$ of inhibition (IC30) was $171 \pm 19.8 \mu \mathrm{g} / \mathrm{mL}$ with a TE of $88.7 \pm 7.7$ Trolox $\mu \mathrm{mol} / \mathrm{g}$ lipid. The polar lipid-rich extract concentration providing $50 \%$ inhibition (IC50) in the ABTS assay was $26.2 \pm 0.1 \mu \mathrm{g} / \mathrm{mL}$ with a TE of $555.4 \pm 28.1$ Trolox $\mu \mathrm{mol} / \mathrm{g}$ lipid. The reactivity and the capacity of the extract rich in polar lipids to scavenge these radical were very different. While for the ABTS assay it was possible to calculate $50 \%$ inhibition, in the case of the DPPH assay, the maximum percentage of inhibition reached nearly $30 \%$.

\section{Discussion}

The consumption of long-chain PUFA, particularly $n-3$, may have long-term health benefits. Their positive effects include reduced cardiovascular disease, morbidity, and mortality [30-32]; enhanced 
visual and neurological development; and improved of inflammatory conditions, such as arthritis and asthma [33-35]. It is recognized that seafood is the main source of $n-3$ long-chain PUFAs, mainly EPA and docosahexaenoic acid (DHA). However, global overexploitation and depletion of marine fish stocks is of long-standing concern, as existing populations of wild and farmed species are unlikely sufficient to meet $n-3$ PUFA requirements for human consumption. In this context, P. palmata appears as an interesting source of EPA that can be supplied in a socio-ecologically sustainable way. Namely its production in IMTA systems compared to current wild harvesting practices might ensure the availability of biomass with increased stability of its nutritional value and biochemical profile, namely its polar lipids and its PUFA composition [36,37].

As described above (Tables 1-4), PL and GL of P. palmata are quite rich in EPA. Our work is consistent with previous studies that reported high levels of EPA in P. palmata compared to other edible seaweeds $[8,38]$.

Phospholipids, in particular those rich in PUFA (namely EPA), contribute significantly to the added value of P. palmata. There is evidence that PLs are better delivers of PUFA than triglycerides (TGs) $[39,40]$. Several studies suggested that much of the dietary PLs fraction is integrated into high-density lipoprotein (HDL) in the intestine, later joining the plasma HDL pool [41-43]. Since PLs are associated with low toxicity, allowing their use for any route of administration, PL from P. palmata are potential candidates for food and nutraceutical formulations.

Phosphatidylcholine is the most abundant phospholipid class of P. palmata. Administration of PCs has been reported to be beneficial for senescence, cognitive function, inflammatory diseases, and plasma and hepatic lipid metabolism [44-47]. It can also be used as a choline supplier. Choline is a component of the vitamin B complex, essential for human nutrition and used by the body to produce acetylcholine, one of the major neurotransmitters of the nervous system, involved in neural networks associated with memory [48]. Choline supplementation is important for vegans and vegetarians, who are at a higher risk for choline deficiency. Some studies also linked high choline intake with reduced risk of breast and colorectal cancer [49-51]. Thus P. palmata PLs could be considered as a potential functional food or as an ingredient for the fortification of foods for nutraceutical applications.

Other potential uses of P. palmata PLs are either in the cosmetics or in the pharma industries. Cosmetic formulations use the emulsifying properties of PLs for skin moisturizing products [52]. Pharmaceutical applications of PLs rely on their ability to form liposomes after mixing in aqueous media, making them a potential drug delivery vector $[52,53]$. Therefore, P. palmata PLs can potentially be used as emulsifiers, as an $n-3$ FAs supplement, and as beneficial nutritional biomolecules. Macroalgae are also considered a promising source of value-added phytochemicals. Several studies have reported that glycolipids can also display bioactive activities, including antioxidant [54], anti-inflammatory [14], anti-proliferative [55], and anti-microbial [56] activities. Some of the glycolipids identified in P. palmata have already been described as having bioactive properties, such as SQDG(34:2) with antiviral activity against HCM-virus [16], SQMD(16:0) with antibacterial activity against Xanthomonas oryzae [57], and the SQDG carrier of EPA with anti-proliferative effect on the inhibition of human telomerase [12] and DNA polymerase $\alpha$ and $\beta$ [58]. Additionally, a study by Banskota and collaborators [14] attributed the anti-inflammatory action of $P$. palmata to polar lipids, including SQDG(20:5;14:0), via the inhibitory activity of nitric oxide, highlighting the role of EPA. Moreover, a MGDG carrier of EPA was patented because of its anti-inflammatory action [59]. The search for natural antioxidants has long attracted the attention of researchers, since inflammation is associated with oxidative stress occurring in several diseases, including chronic and age-related ones [60,61]. Oxidative stress occurs when the production of radicals increases and exceeds the detoxification capacity of cellular antioxidant defense systems. Antioxidants are biomolecules that prevent, or delay damage to other molecules, by the presence of free radicals, because they have the ability to block or inhibit them directly or indirectly, or because they stimulate cellular antioxidant defenses [62]. Antioxidants from natural or organic sources aim not only to promote the development of new drugs but also to act in the prevention of inflammatory diseases [63]. Such biomolecules with antioxidant properties can be obtained from foods, including 
plants and marine vegetables $[64,65]$. There are some studies of seaweed lipid characterization using lipidomics based on the LC-MS approach that allowed the putative bioactivity and health benefits of seaweeds lipids to be highlighted, as well the exploitation of seaweeds as a functional food, as reported for Codium tomentosum, Porphyra dioica, Gracilaria sp, Chondrus crispus, Ulva rigida, Saccharina latissima, and Fucus vesiculosus [21-25,66,67]. However, seaweeds' lipids antioxidant potential was not explored.

The antioxidant activity of seaweeds has been mostly related to their phenolic compounds and pigment contents [68-72], whereas it is only now that lipids are beginning to deserve special attention. To the best of our knowledge, there is only one study of lipids' fraction antioxidant activity for the red and brown seaweeds Solieria chordalis ((C.Agardh) J. Agardh, 1842) and Sargassum muticum ((Yendo) Fensholt, 1955), respectively, whose antioxidant potential was lower than the one obtained in this study for P. palmata [19]. Overall, our results showed that the polar lipid-rich extract of P. palmata exhibits antioxidant proprieties, showing a higher percentage inhibition of DPPH and TE than those of the methanolic extract of P. palmata, mainly composed of phenolic compounds, as previously described [68]. It also showed significantly better antioxidant activity for the $\mathrm{DPPH}^{\bullet}$ and $\mathrm{ABTS}^{\bullet+}$ assays compared to the red seaweed Gracilaria manilaensis (Yamamoto \& Trono, 1994) organic extract [73]. The polar lipid-rich extract of P. palmata still had a higher antioxidant potential for the $\mathrm{ABTS}^{\bullet+}$ assay compared to a study of antioxidant activity using different organic solvents with phenolic content from the brown seaweed Sargassum serratifolium ((C. Agardh) C. Agardh, 1820) [74].

\section{Materials and Methods}

\subsection{Reagents}

Phospholipid standards 1,2-dimyristoyl-sn-glycero-3-phosphocholine (dMPC), 1,2-dimyristoyl-snglycero-3-phosphoethanolamine (dMPE), 1,2-dimyristoyl-sn-glycero-3-phospho-(1'-rac-)glycerol (dMPG), 1,2-dimyristoyl-sn-glycero-3-phospho-L-serine (dMPS), 1',3'-bis[1-dimyristoyl-sn-glycero-3phospho]-glycerol (tMCL), 1,2-dipalmitoyl-sn-glycero-3-phosphatidylinositol (dPPI), N-palmitoylD-erythro-sphingosylphosphorylcholine (NPSM), and 1-nonadecanoyl-2-hydroxy-sn-glycero3-phosphocholine (LPC) were purchased from Avanti Polar Lipids, Inc. (Alabaster, AL). Chloroform $\left(\mathrm{CHCl}_{3}\right)$, methanol $(\mathrm{MeOH})$, ethanol absolute, and acetonitrile were purchased from Fisher scientific (Leicestershire, UK); all the solvents were of high-performance liquid chromatography (HPLC) grade and were used without further purification. $\mathrm{DPPH}^{\bullet}$ was purchased from Aldrich (Milwaukee, WI). 2,20-Azino-bis(3-ethylbenzothiazoline-6-sulfonic acid) diammonium salt $\left(\mathrm{ABTS}^{\bullet+}\right.$ ) was obtained from Fluka (Buchs, Switzerland). Ammonium acetate and 6-hydroxy-2,5,7,8-tetramethylchromane-2-carboxylic acid (Trolox) were purchased from Sigma-Aldrich (St Louis, MO, USA). All the other reagents and chemicals used were of the highest grade of purity commercially available. Milli-Q water was also used (Synergysup ${ }^{\circledR}$, Millipore Corporation, Billerica, MA, USA).

\subsection{Seaweed Biomass}

The biomass of Palmaria palmata ((Linnaeus) F.Weber \& D.Mohr, 1805) was provided by a local aquaculture producer-ALGAplus-who farms seaweed integrated with finfish production (located at Ria de Aveiro coastal lagoon, mainland Portugal, $40^{\circ} 36^{\prime} 43^{\prime \prime} \mathrm{N}, 8^{\circ} 40^{\prime} 43^{\prime \prime} \mathrm{W}$ ). Palmaria palmata is produced by vegetative propagation, in a controlled indoor system from June to September (approximately) and in an open-flow outdoor tank system during colder months. For this work, the red algae were harvested in May 2018, cleaned to remove the epiphytes, and washed using sterilized seawater. It was then dried at $25{ }^{\circ} \mathrm{C}$ in an air tunnel until $10 \%$ to $12 \%$ total moisture was reached. Five $250-\mathrm{mg}$ aliquots were obtained from bulk production and were used for total lipid extraction.

\subsection{Lipid Extraction}

The biomass of P. palmata was cut into small pieces and ground in a mortar and pestle with liquid nitrogen until homogenized. The lipid extraction procedure was performed using a modified Bligh 
and Dyer protocol [23], mixing $250 \mathrm{mg}$ of seaweed biomass (five replicates) with $2.5 \mathrm{~mL}$ of $\mathrm{MeOH}$ and $1.25 \mathrm{~mL}$ of $\mathrm{CHCl}_{3}$ in a glass PYREX tube and homogenized by vortexing for $2 \mathrm{~min}$ and incubation in ice on a rocking platform shaker (Stuart equipment, Bibby Scientific, Stone, UK) for $2 \mathrm{~h}$ and $30 \mathrm{~min}$. The mixture was centrifuged (Selecta JP Mixtasel, Abrera, Barcelona, Spain) for $10 \mathrm{~min}$ at $2000 \mathrm{rpm}$ and the organic phase was collected in a new glass tube. The biomass residue was re-extracted twice with $2 \mathrm{~mL}$ of $\mathrm{MeOH}$ and $1 \mathrm{~mL}$ of $\mathrm{CHCl}_{3}$. To wash the lipid extract and induce phase separation, $2.3 \mathrm{~mL}$ of Milli-Q water was added to the final organic phase, followed by centrifugation for $10 \mathrm{~min}$ at $2000 \mathrm{rpm}$. The organic lower phase was collected in a new glass tube and dried under a nitrogen stream. Lipid extracts were then transferred to amber vials, dried again, weighed, and stored at $-20^{\circ} \mathrm{C}$. Lipid content was estimated as a dry weight percentage.

\subsection{Fatty Acid Analysis by Gas Chromatography Mass Spectrometry}

Fatty acid methyl esters (FAMEs) were prepared using a methanolic solution of potassium hydroxide $(2.0 \mathrm{M})$ (Melo et al., 2015). A sample volume of $2 \mu \mathrm{L}$ of hexane solution containing FAMEs was analyzed by gas chromatography mass spectrometry (GC-MS) on a GC system (Agilent Technologies 6890 N Network, Santa Clara, CA, USA) equipped with a DB-FFAP column with the following specifications: $30 \mathrm{~m}$ long, $0.32 \mathrm{~mm}$ internal diameter, and $0.25 \mu \mathrm{m}$ film thickness (123-3232, J \& W Scientific, Folsom, CA, USA). The GC equipment was connected to an Agilent 5973 Network Mass Selective Detector operating with an electron impact mode at $70 \mathrm{eV}$ and scanning range $\mathrm{m} / \mathrm{z}$ of 50 to 550 in a one second cycle in full scan mode acquisition. The oven temperature was programmed from an initial temperature of $80^{\circ} \mathrm{C}$ for $3 \mathrm{~min}$, a linear increase to $160^{\circ} \mathrm{C}$ at $25^{\circ} \mathrm{C} \mathrm{min}-1$, followed by linear increase at $2^{\circ} \mathrm{C} \mathrm{min}^{-1}$ to $210^{\circ} \mathrm{C}$, then at $30^{\circ} \mathrm{C} \mathrm{min}-1$ to $250{ }^{\circ} \mathrm{C}$, standing at $250{ }^{\circ} \mathrm{C}$ for $10 \mathrm{~min}$. The injector and detector temperatures were 220 and $280^{\circ} \mathrm{C}$, respectively. Helium was used as the carrier gas at a flow rate of $1.4 \mathrm{~mL} \mathrm{~min}^{-1}$. FA identification was performed considering the retention times and MS spectra of FA standards (Supelco 37 Component Fame Mix, Sigma-Aldrich), and by MS spectrum comparison with chemical databases (Wiley 275 library and AOCS lipid library). FAMEs of five analytical replicates were injected. The relative amounts of FAs were calculated by the percent relative area method with proper normalization using internal standard methyl nonadecanoate (C19:0, Sigma-Aldrich, St. Louis, MO, USA), considering the sum of all relative areas of identified FAs.

\subsection{Polar Lipid Analysis by Hydrophilic Interaction Liquid Chromatography Mass Spectrometry (HILIC-ESI-MS)}

Lipid extracts were analyzed by mass spectrometry using hydrophilic interaction liquid chromatography (HILIC) on an Ultimate 3000 Dionex (Thermo Fisher Scientific, Bremen, Germany) with an autosampler coupled to a Q-Exactive hybrid quadrupole mass spectrometer (Thermo Fisher, Scientific, Bremen, Germany). The elution method was previously described $[21,24,25]$ and applied with some modifications. The system is based on two mobile phases: Mobile phase A ( $25 \%$ water, $50 \%$ acetonitrile, and $25 \%$ methanol) and mobile phase B ( $60 \%$ acetonitrile and $40 \%$ methanol), both with $1 \mathrm{mM}$ ammonium acetate. Firstly, $40 \%$ of mobile phase A was held isocratically for $8 \mathrm{~min}$, followed by a linear increase to $60 \%$ of mobile phase A within $7 \mathrm{~min}$, and maintained for $5 \mathrm{~min}$. After that, conditions returned to the initial settings in $15 \mathrm{~min}$ (5 min to decrease to $40 \%$ of phase A and a re-equilibration period of $10 \mathrm{~min}$ prior to the next injection). A volume of $5 \mu \mathrm{L}$ of each sample, containing $5 \mu \mathrm{g}$ of lipid extract in $\mathrm{CHCl}_{3}, 4 \mu \mathrm{L}$ of phospholipid standards mix (dMPC- $0.02 \mu \mathrm{g}$,

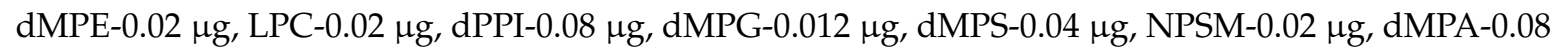
$\mu \mathrm{g}$, tMCL- $0.08 \mu \mathrm{g})$, and $91 \mu \mathrm{L}$ of starting eluent ( $60 \% \mathrm{~B}$ and $40 \% \mathrm{~A})$, was introduced into the Ascentis Si column HPLC Pore column ( $15 \mathrm{~cm} \times 1 \mathrm{~mm}, 3 \mu \mathrm{m}$, Sigma-Aldrich) with a flow rate of $40 \mu \mathrm{L} \mathrm{min}{ }^{-1}$ at $30^{\circ} \mathrm{C}$. The mass spectrometer with Orbitrap ${ }^{\circledR}$ technology was operated in simultaneous positive (electrospray voltage $3.0 \mathrm{kV}$ ) and negative (electrospray voltage $-2.7 \mathrm{kV}$ ) modes with high resolution with 70,000 and AGC target of $1 \times 10^{6}$, the capillary temperature was $250{ }^{\circ} \mathrm{C}$, and the sheath gas flow was $15 \mathrm{U}$. The tandem mass spectrometry experiments were performed at a resolution of 17,500 
and AGC target of $1 \times 10^{5}$ with one full scan mass spectrum and 10 data-dependent MS/MS scans. The cycles were repeated continuously throughout the experiments with the dynamic exclusion of $60 \mathrm{~s}$ and an intensity threshold of $1 \times 10^{4}$. Normalized collision energy ${ }^{\mathrm{TM}}(\mathrm{CE})$ ranged between 25 , 30, and $35 \mathrm{eV}$. Data acquisition was performed using the Xcalibur data system (V3.3, Thermo Fisher Scientific, USA). The identification of molecular species of polar lipids was based on the assignment of the molecular ions observed in the LC-MS spectra, typical retention time, mass accuracy, and MS/MS spectra information.

\subsection{Data Analysis}

In a first approach, the lipid species of P. palmata were identified using MZmine 2.32 software. This tool allows the analysis of raw data acquired in full MS by the integration of each lipid species peak, peak processing, and assignment against an in-house database. The validated peaks were within the time range of a MS full run and peaks with raw intensity lower than 1e4 were excluded. All the information originating from the MZmine software was confirmed based on the assignment of the molecular ions observed in the LC-MS spectra, typical retention time, exact mass accuracy, and MS/MS spectra information. Only exact mass accuracy with an error of less than $5 \mathrm{ppm}$ was considered. The typical fragmentation of each lipid class in MS/MS spectra was taken into account according to what has already been described in the literature [25]. The normalization of the identified lipid species was performed by exporting integrated peak areas values (.csv file) and dividing the peak area value of each species by the peak area value of a standard lipid species with the closest retention time. The lipid species' relative abundance was calculated in terms of the percentage dividing the normalized value of each lipid species by the sum of all identified lipid species. Bar graphs were created using the software GraphPad Prism 8.0.1

\subsection{2-Diphenyl-1-Picrylhydrazyl Radical Assay—DPPH Radical Scavenging Activity}

The antioxidant scavenging activity against the $\alpha, \alpha$-diphenyl- $\beta$-picrylhydrazyl radical (DPPH ${ }^{\bullet}$ ) was evaluated using a previously described method $[75,76]$ applied with some modifications. A stock solution of $\mathrm{DPPH}^{\bullet}$ in ethanol $(250 \mu \mathrm{mol} / \mathrm{L})$ was prepared and diluted to provide a working solution with an absorbance value of $\sim 0.9$ measured at $517 \mathrm{~nm}$ using a UV-vis spectrophotometer (Multiskan GO 1.00.38, Thermo Scientific, Hudson, NH, USA) controlled by the SkanIT software version 3.2 (Thermo Scientific). To evaluate the radical stability, a volume of $150 \mu \mathrm{L}$ of ethanol was added to 15 microplate wells followed by addition of $150 \mu \mathrm{L}$ of $\mathrm{DPPH}^{\bullet}$ diluted solution and an incubation period of $120 \mathrm{~min}$, with absorbance measured at $517 \mathrm{~nm}$ every $5 \mathrm{~min}$. For evaluation of the radical scavenging potential, a volume of $150 \mu \mathrm{L}$ of $P$. palmata lipid extract $(25,50,100,250 \mu \mathrm{g} / \mathrm{mL}$ in ethanol) and $150 \mu \mathrm{L}$ of Trolox standard solution $(5,12.5,25,37.5 \mu \mathrm{mol} / \mathrm{L}$ in ethanol) were placed in each well followed by addition of $150 \mu \mathrm{L}$ of $\mathrm{DPPH}^{\bullet}$ diluted solution, and again an incubation period of $120 \mathrm{~min}$, with absorbance measured at $517 \mathrm{~nm}$ every $5 \mathrm{~min}$. The control lipid extracts were also assayed by replacing $150 \mu \mathrm{L}$ of $\mathrm{DPPH}^{\bullet}$ diluted solution by $150 \mu \mathrm{L}$ of ethanol. Radical reduction by hydrogen donor antioxidants was monitored by measuring the decrease in absorbance during the reaction, thereby quantifying radical scavenging, which is accompanied by a radical color change. All measurements were performed in triplicate on two different days. The \% of DPPH radical remaining was determined according to:

$\%$ DPPH remaining $=($ Abs samples after $120 \mathrm{~min} /$ Abs sample at the beginning of reaction $) \times 100$.

The free radical-scavenging activity of samples was determined as the percentage of inhibition of DPPH radical according to:

Inhibition $\%=((A b s$ DPPH $-($ Abs samples - Abs control $)) / A b s D P P H) \times 100$. 
The concentration of samples capable of reducing 30\% of DPPH radical after $120 \mathrm{~min}$ (IC30) were calculated by linear regression using the concentration of samples and the percentage of the inhibition curve. The activity is expressed as Trolox Equivalents (TE, $\mu \mathrm{mol}$ Trolox/g of sample), according to:

$$
\text { TE = IC30 Trolox }(\mu \mathrm{mol} / \mathrm{L}) \times 1000 / I C 30 \text { of samples }(\mu \mathrm{g} / \mathrm{mL}) \text {. }
$$

\subsection{2,20-Azino-bis-3-Ethylbenzothiazoline-6-Sulfonic Acid Radical Cation Assay-ABTS Radical Scavenging Activity}

The antioxidant scavenging activity against the 2,20-azino-bis-3-ethylbenzothiazoline-6-sulfonic acid radical cation $\left(\mathrm{ABTS}^{\bullet+}\right.$ ) was evaluated using a previously described method [76,77] applied with some modifications. The ABTS radical solution $(3.5 \mathrm{mmol} / \mathrm{L})$ was prepared by mixing $10 \mathrm{~mL}$ of ABTS stock solution $\left(7 \mathrm{mmol} / \mathrm{L}\right.$ in water) with $10 \mathrm{~mL}$ of potassium persulfate $\mathrm{K}_{2} \mathrm{~S}_{2} \mathrm{O}_{8}(2.45 \mathrm{mmol} / \mathrm{L}$ in water) $[77,78]$. This mixture was kept for $12 \mathrm{~h}$ at room temperature and was diluted in ethanol to obtain an absorbance value of $\sim 0.9$ measured at $734 \mathrm{~nm}$ using a UV-vis spectrophotometer (Multiskan GO 1.00.38, Thermo Scientific, Hudson, NH, USA) controlled by the SkanIT software version 3.2 (Thermo Scientific). For an evaluation of the radical stability, a volume of $150 \mu \mathrm{L}$ of ethanol was added to 15 microplate wells followed by addition of $150 \mu \mathrm{L}$ of $\mathrm{ABTS}^{\bullet+}$ diluted solution and an incubation period of $120 \mathrm{~min}$, with absorbance measured at $734 \mathrm{~nm}$ every $5 \mathrm{~min}$. For an evaluation of the radical scavenging potential, a volume of $150 \mu \mathrm{L}$ of lipid extract $(25,50,100,250 \mu \mathrm{g} / \mathrm{mL}$ in ethanol) and $150 \mu \mathrm{L}$ of Trolox standard solution $(4,8,16,28,40,56 \mu \mathrm{mol} / \mathrm{L}$ in ethanol) were placed in each well followed by addition of $150 \mu \mathrm{L}$ of $\mathrm{ABTS}^{\bullet+}$ diluted solution, and a new incubation period of $120 \mathrm{~min}$, with absorbance measurements at $734 \mathrm{~nm}$ every $5 \mathrm{~min}$. The control lipid extracts were also assayed by replacing $150 \mu \mathrm{L}$ of ABTS $^{\bullet+}$ diluted solution by $150 \mu \mathrm{L}$ of ethanol. Radical reduction by hydrogen donor antioxidants was monitored by measuring the decrease in absorbance during the reaction, thereby quantifying radical scavenging, which is accompanied by a radical color change. All measurements were performed in triplicate on two different days. The \% of ABTS radical remaining was determined according to:

$$
\% \text { ABTS remaining }=(\text { Abs samples after } 120 \mathrm{~min} / \text { Abs sample at the beginning of reaction }) \times 100 \text {. }
$$

The free radical-scavenging activity of samples was determined as the percentage of inhibition of ABTS radical according to:

$$
\text { Inhibition } \%=((\text { Abs ABTS }-(\text { Abs samples }- \text { Abs control })) / \text { Abs ABTS }) \times 100 .
$$

The concentration of samples capable of reducing 30\% of ABTS radical after 120 min (IC50) were calculated by linear regression using the concentration of samples and the percentage of the inhibition curve. The activity is expressed as Trolox Equivalents (TE, $\mu \mathrm{mol}$ Trolox/g of sample), according to:

$$
\text { TE }=\text { IC50 Trolox }(\mu \mathrm{mol} / \mathrm{L}) \times 1000 / I C 50 \text { of samples }(\mu \mathrm{g} / \mathrm{mL}) \text {. }
$$

\section{Conclusions}

In this study, the use of a mass spectrometry-based approach identified 144 lipid species of P. palmata produced in an environmentally friendly IMTA system. Eicosapentaenoic acid was the most abundant fatty acid identified in P. palmata, with a relative abundance greater than $50 \%$. The content of EPA may be one of the major factors in the antioxidant activity of the polar lipid-rich extract, which has been found to be superior to that of phenolic compounds described in previous studies. Nevertheless, our results may not be exclusive to lipids, and synergy with other molecules should be taken into account. These findings revealed the lipid profile of P. palmata and highlighted that when farmed under IMTA, P. palmata can be a stable and sustainable source of beneficial lipids. The findings reported in this study add value to the polar lipid-rich extract of $P$. palmata and open a window of opportunity for 
innovative biotechnological applications targeting algal-based products. This work is the beginning of polar lipid prospection in P. palmata biomass and its results should now be validated across other possible P. palmata sources.

Author Contributions: Conceptualization, M.R.D., T.M. and D.L.; methodology and formal analysis, D.L., J.M. and T.M.; validation, T.M. and M.R.D.; investigation, D.L. and J.M.; writing original draft preparation, D.L. and M.R.D.; resources, M.H.A., R.P., M.R.D., P.D., R.C. and A.L.; writing, review and editing, all authors.

Funding: The authors are grateful to ALGAplus- Produção e Comércio de algas e seus derivados, Lda. for supplying the seaweed samples. This research was funded by Fundação para a Ciência e a Tecnologia (FCT, Portugal), European Union, QREN, POPH, FEDER, and COMPETE by funding QOPNA-LAQV research unit (FCT UID/QUI/ 00062/2019), RNEM (LISBOA-01-0145-FEDER-402-022125) for the Portuguese Mass Spectrometry Network, CESAM (UID/AMB/50017/2019) financed by Portuguese funds through the FCT/MEC, and when applicable co-financed by FEDER under the PT2020 Partnership Agreement and project Omics 4 Algae: Lipidomic tools for chemical phenotyping, traceability and valorisation of seaweeds from aquaculture as a sustainable source of high added-value compounds (POCI-01-0145-FEDER-030962), funded by Centro2020, through FEDER and PT2020. Diana Lopes (SFRH/BD/119027/2016) is grateful to FCT (Fundação para a Ciência e Tecnologia), Programa Operacional do Capital Humano (POCH) and European Union through European Social Fund (FSE) for her grant. Tânia Melo thanks the research contract under the project Omics 4 Algae: Lipidomic tools for chemical phenotyping, traceability and valorisation of seaweeds from aquaculture as a sustainable source of high added-value compounds (POCI-01-0145-FEDER-030962), funded by Centro2020, through FEDER and PT2020. This work is a contribution of the Marine Lipidomics Laboratory and was also supported by the Integrated Programme of SmartBioR "Smart Valorization of Endogenous Marine Biological Resources Under a Changing Climate" (Centro-01-0145-FEDER-000018), co-funded by Centro 2020 program, Portugal 2020, European Union, through the European Regional Development Fund.

Conflicts of Interest: The authors declare no conflict of interest.

\section{References}

1. McHugh, D.J. A Guide to the Seaweed Industry; FAO Fisheries Technical Paper; FAO: Rome, Italy, $2003 ;$ p. 105. ISBN 9-25-104958-0.

2. Bixler, H.J.; Porse, H. A decade of change in the seaweed hydrocolloids industry. J. Appl. Phycol. 2011, 23, 321-335. [CrossRef]

3. Sutherland, J.E.; Lindstrom, S.C.; Nelson, W.A.; Brodie, J.; Lynch, M.D.J.; Hwang, M.S.; Choi, H.; Miyata, M.; Kikuchi, N.; Oliveira, M.C.; et al. A new look at an ancient order: Generic revision of the Bangiales (Rhodophyta). J. Phycol. 2011, 47, 1131-1151. [CrossRef] [PubMed]

4. Cultured Aquatic Species Information Programme. Porphyra spp. Cultured Aquatic Species Information Programme; Chen, J., Xu, P., Eds.; FAO Fisheries and Aquaculture Department: Rome, Italy, 2005.

5. Grote, B. Recent developments in aquaculture of Palmaria palmata (Linnaeus) (Weber \& Mohr 1805): Cultivation and uses. Rev. Aquac. 2019, 11, 25-41.

6. Morgan, K.C.; Wright, J.L.C.; Simpson, F.J. Review of chemical constituents of the red alga Palmaria palmata (dulse). Econ. Bot. 1980, 34, 28-50. [CrossRef]

7. Mishra, V.K.; Temelli, F.; Ooraikul, B.; Shacklock, P.F.; Craigie, J.S. Lipids of the Red Alga, Palmaria palmata. Bot. Mar. 1993, 36, 169-174. [CrossRef]

8. Van Ginneken, V.J.; Helsper, J.P.; de Visser, W.; van Keulen, H.; Brandenburg, W.A. Polyunsaturated fatty acids in various macroalgal species from north Atlantic and tropical seas. Lipids Health Dis. 2011, 10, 1-8. [CrossRef] [PubMed]

9. Borow, K.M.; Nelson, J.R.; Mason, R.P. Biologic plausibility, cellular effects, and molecular mechanisms of eicosapentaenoic acid (EPA) in atherosclerosis. Atherosclerosis 2015, 242, 357-366. [CrossRef]

10. Gdula-Argasińska, J.; Czepiel, J.; Woźniakiewicz, A.; Wojtoń, K.; Grzywacz, A.; Woźniakiewicz, M.; Jurczyszyn, A.; Perucki, W.; Librowski, T. N-3 Fatty acids as resolvents of inflammation in the A549 cells. Pharmacol. Rep. 2015, 67, 610-615. [CrossRef]

11. Meyer, B.J. Are we consuming enough long chain omega-3 polyunsaturated fatty acids for optimal health? Prostaglandins Leukot. Essent. Fat. Acids 2011, 85, 275-280. [CrossRef]

12. Eitsuka, T.; Nakagawa, K.; Igarashi, M.; Miyazawa, T. Telomerase inhibition by sulfoquinovosyldiacylglycerol from edible purple laver (Porphyra yezoensis). Cancer Lett. 2004, 212, 15-20. [CrossRef] 
13. Ohta, K.; Mizushina, Y.; Hirata, N.; Takemura, M.; Sugawara, F.; Matsukage, A.; Yoshida, S.; Sakaguchi, K. Sulfoquinovosyldiacylglycerol, KM043, a new potent inhibitor of eukaryotic DNA polymerases and HIV-reverse transcriptase type 1 from a marine red alga, Gigartina tenella. Chem. Pharm. Bull. (Tokyo) 1998, 46, 684-686. [CrossRef] [PubMed]

14. Banskota, A.H.; Stefanova, R.; Sperker, S.; Lall, S.P.; Craigie, J.S.; Hafting, J.T.; Critchley, A.T. Polar lipids from the marine macroalga Palmaria palmata inhibit lipopolysaccharide-induced nitric oxide production in RAW264.7 macrophage cells. Phytochemistry 2014, 101, 101-108. [CrossRef] [PubMed]

15. Banskota, A.H.; Stefanova, R.; Sperker, S.; Melanson, R.; Osborne, J.A.; O'Leary, S.J.B. Five new galactolipids from the freshwater microalga Porphyridium aerugineum and their nitric oxide inhibitory activity. J. Appl. Phycol. 2013, 25, 951-960. [CrossRef]

16. El Baz, F.K.; El Baroty, G.S.; Abd El Baky, H.H.; Abd El Salam, O.I.; Ibrahim, E.A. Structural characterization and Biological Activity of Sulfolipids from selected Marine Algae. Grasas y Aceites 2013, 64, 561-571.

17. Parveez, A.A.; Rasheed, U.M.; Noorani, K.P.M.; Reehana, N.; Santhoshkumar, S.; Imran, Y.M.M.; Alharbi, S.N.; Arunachalam, C.; Alharbi, A.S.; Akbarsha, M.A.; et al. In vitro antibacterial activity of MGDG-palmitoyl from Oscillatoria acuminata NTAPC05 against extended-spectrum $\beta$-lactamase producers. J. Antibiot. (Tokyo) 2017, 70, 754-762. [CrossRef] [PubMed]

18. Wang, H.; Li, Y.L.; Shen, W.Z.; Rui, W.; Ma, X.J.; Cen, Y.Z. Antiviral activity of a sulfoquinovosyldiacylglycerol (SQDG) compound isolated from the green alga Caulerpa racemosa. Bot. Mar. 2007, 50, 185-190. [CrossRef]

19. Terme, N.; Boulho, R.; Kucma, J.-P.; Bourgougnon, N.; Bedoux, G. Radical scavenging activity of lipids from seaweeds isolated by solid-liquid extraction and supercritical fluids. OCL 2018, 25, D505. [CrossRef]

20. Chakraborty, K.; Maneesh, A.; Makkar, F. Antioxidant Activity of Brown Seaweeds. J. Aquat. Food Prod. Technol. 2017, 26, 406-419. [CrossRef]

21. Da Costa, E.; Melo, T.; Moreira, A.S.P.; Alves, E.; Domingues, P.; Calado, R.; Abreu, M.H.; Domingues, M.R. Decoding bioactive polar lipid profile of the macroalgae Codium tomentosum from a sustainable IMTA system using a lipidomic approach. Algal Res. 2015, 12, 388-397. [CrossRef]

22. Da Costa, E.; Azevedo, V.; Melo, T.; Rego, A.M.; Evtuguin, D.V.; Domingues, P.; Calado, R.; Pereira, R.; Abreu, M.H.; Domingues, M.R. High-Resolution Lipidomics of the Early Life Stages of the Red Seaweed Porphyra dioica. Molecules 2018, 23, 187. [CrossRef]

23. Da Costa, E.; Melo, T.; Moreira, A.S.P.; Bernardo, C.; Helguero, L.; Ferreira, I.; Cruz, M.T.; Rego, A.M.; Domingues, P.; Calado, R.; et al. Valorization of Lipids from Gracilaria sp. through Lipidomics and Decoding of Antiproliferative and Anti-Inflammatory Activity. Mar. Drugs 2017, 15, 62. [CrossRef] [PubMed]

24. Melo, T.; Alves, E.; Azevedo, V.; Martins, A.S.; Neves, B.; Domingues, P.; Calado, R.; Abreu, H.; Domingues, M.R. Lipidomics as a new approach for the bioprospecting of marine macroalgae - unraveling the polar lipid and fatty acid composition of Chondrus crispus. Algal Res. 2015, 8, 181-191. [CrossRef]

25. Lopes, D.; Moreira, A.S.P.; Rey, F.; da Costa, E.; Melo, T.; Maciel, E.; Rego, A.; Abreu, M.H.; Domingues, P.; Calado, R.; et al. Lipidomic signature of the green macroalgae Ulva rigida farmed in a sustainable integrated multi-trophic aquaculture. J. Appl. Phycol. 2019, 31, 1369-1381. [CrossRef]

26. Byju, K.; Vasundhara, G.; Anuradha, V.; Nair, S.M.; Kumar, N.C. Presence of Phytol, a Precursor of Vitamin E in Chaetomorpha Antinnina. Mapana-J. Sci. 2013, 12, 57-65. [CrossRef]

27. Guschina, I.A.; Harwood, J.L. Lipids and lipid metabolism in eukaryotic algae. Prog. Lipid Res. 2006, 45, 160-186. [CrossRef] [PubMed]

28. Li-Beisson, Y.; Thelen, J.J.; Fedosejevs, E.; Harwood, J.L. The lipid biochemistry of eukaryotic algae. Prog. Lipid Res. 2019, 74, 31-68. [CrossRef] [PubMed]

29. Petroutsos, D.; Amiar, S.; Abida, H.; Dolch, L.J.; Bastien, O.; Rébeillé, F.; Jouhet, J.; Falconet, D.; Block, M.A.; McFadden, G.I.; et al. Evolution of galactoglycerolipid biosynthetic pathways-From cyanobacteria to primary plastids and from primary to secondary plastids. Prog. Lipid Res. 2014, 54, 68-85. [CrossRef] [PubMed]

30. Huang, H.-L.; Wang, B.-G. Antioxidant capacity and lipophilic content of seaweeds collected from the Qingdao coastline. J. Agric. Food Chem. 2004, 52, 4993-4997. [CrossRef]

31. Mozaffarian, D.; Ascherio, A.; Hu, F.B.; Stampfer, M.J.; Willett, W.C.; Siscovick, D.S.; Rimm, E.B. Interplay between different polyunsaturated fatty acids and risk of coronary heart disease in men. Circulation 2005, 111, 157-164. [CrossRef] 
32. Marik, P.E.; Varon, J. Omega-3 dietary supplements and the risk of cardiovascular events: A systematic review. Clin. Cardiol. 2009, 32, 365-372. [CrossRef]

33. SanGiovanni, J.P.; Parra-Cabrera, S.; Colditz, G.A.; Berkey, C.S.; Dwyer, J.T. Meta-analysis of Dietary Essential Fatty Acids and Long-Chain Polyunsaturated Fatty Acids as They Relate to Visual Resolution Acuity in Healthy Preterm Infants. Pediatrics 2004, 105, 1292-1298. [CrossRef] [PubMed]

34. Rennie, K.L.; Hughes, J.; Lang, R.; Jebb, S.A. Nutritional management of rheumatoid arthritis: A review of the evidence. J. Hum. Nutr. Diet. 2003, 16, 97-109. [CrossRef] [PubMed]

35. Barros, R.; Moreira, A.; Fonseca, J.; Delgado, L.; Graça Castel-Branco, M.; Haahtela, T.; Lopes, C.; Moreira, P. Dietary intake of $\alpha$-linolenic acid and low ratio of n -6: N -3 PUFA are associated with decreased exhaled NO and improved asthma control. Br. J. Nutr. 2011, 106, 441-450. [CrossRef] [PubMed]

36. Chopin, T.; Cooper, J.A.; Reid, G.; Cross, S.; Moore, C. Open-water integrated multi-trophic aquaculture: Environmental biomitigation and economic diversification of fed aquaculture by extractive aquaculture. Rev. Aquac. 2012, 4, 209-220. [CrossRef]

37. Paiva, L.; Lima, E.; Neto, A.I.; Baptista, J. Seasonal Variability of the Biochemical Composition and Antioxidant Properties of Fucus spiralis at Two Azorean Islands. Mar. Drugs 2018, 16, 248. [CrossRef] [PubMed]

38. Sánchez-Machado, D.I.; López-Cervantes, J.; López-Hernández, J.; Paseiro-Losada, P. Fatty acids, total lipid, protein and ash contents of processed edible seaweeds. Food Chem. 2004, 85, 439-444. [CrossRef]

39. Lagarde, M.; Bernoud, N.; Brossard, N.; Lemaitre-Delaunay, D.; Thiès, F.; Croset, M.; Lecerf, J. Lysophosphatidylcholine as a preferred carrier form of docosahexaenoic acid to the brain. J. Mol. Neurosci. 2001, 16, 201-204. [CrossRef]

40. Picq, M.; Chen, P.; Perez, M.; Michaud, M.; Véricel, E.; Guichardant, M.; Lagarde, M. DHA Metabolism: Targeting the Brain and Lipoxygenation. Mol. Neurobiol. 2010, 42, 48-51. [CrossRef]

41. Krimbou, L.; Hajj Hassan, H.; Blain, S.; Rashid, S.; Denis, M.; Marcil, M.; Genest, J. Biogenesis and speciation of nascent apoA-I-containing particles in various cell lines. J. Lipid Res. 2005, 46, 1668-1677. [CrossRef]

42. Magun, A.M.; Mish, B.; Glickman, R.M. Intracellular apoA-I and apoB distribution in rat intestine is altered by lipid feeding. J. Lipid Res. 1988, 29, 1107-1116.

43. Wang, H.; Du, J.; Lu, S.; Yao, Y.; Hunter, F.; Black, D.D. Regulation of intestinal apolipoprotein A-I synthesis by dietary phosphatidylcholine in newborn swine. Lipids 2001, 36, 683-687. [CrossRef] [PubMed]

44. Hung, M.-C.; Shibasaki, K.; Yoshida, R.; Sato, M.; Imaizumi, K. Learning behaviour and cerebral protein kinase $\mathrm{C}$, antioxidant status, lipid composition in senescence-accelerated mouse: Influence of a phosphatidylcholine-vitamin B12 diet. Br. J. Nutr. 2001, 86, 163-171. [CrossRef] [PubMed]

45. Schneider, H.; Braun, A.; Füllekrug, J.; Stremmel, W.; Ehehalt, R. Lipid Based Therapy for Ulcerative Colitis-Modulation of Intestinal Mucus Membrane Phospholipids as a Tool to Influence Inflammation. Int. J. Mol. Sci. 2010, 11, 4149-4164. [CrossRef] [PubMed]

46. Chung, S.-Y.; Moriyama, T.; Uezu, E.; Uezu, K.; Hirata, R.; Yohena, N.; Masuda, Y.; Kokubu, Y.; Yamamoto, S. Administration of Phosphatidylcholine Increases Brain Acetylcholine Concentration and Improves Memory in Mice with Dementia. J. Nutr. 1995, 125, 1484-1489. [PubMed]

47. Buang, Y.; Wang, Y.-M.; Cha, J.-Y.; Nagao, K.; Yanagita, T. Dietary phosphatidylcholine alleviates fatty liver induced by orotic acid. Nutrition 2005, 21, 867-873. [CrossRef] [PubMed]

48. Deiana, S.; Platt, B.; Riedel, G. The cholinergic system and spatial learning. Behav. Brain Res. 2011, 221, 389-411. [CrossRef] [PubMed]

49. Xu, X.; Gammon, M.D.; Zeisel, S.H.; Bradshaw, P.T.; Wetmur, J.G.; Teitelbaum, S.L.; Neugut, A.I.; Santella, R.M.; Chen, J. High intakes of choline and betaine reduce breast cancer mortality in a population-based study. FASEB J. 2009, 23, 4022-4028. [CrossRef]

50. Xu, X.; Gammon, M.D.; Zeisel, S.H.; Lee, Y.L.; Wetmur, J.G.; Teitelbaum, S.L.; Bradshaw, P.T.; Neugut, A.I.; Santella, R.M.; Chen, J. Choline metabolism and risk of breast cancer in a population-based study. FASEB J. 2008, 22, 2045-2052. [CrossRef]

51. Lee, J.E.; Giovannucci, E.; Fuchs, C.S.; Willett, W.C.; Zeisel, S.H.; Cho, E. Choline and betaine intake and the risk of colorectal cancer in men. Cancer Epidemiol. Biomark. Prev. 2010, 19, 884-887. [CrossRef]

52. Burri, L.; Hoem, N.; Banni, S.; Berge, K. Marine Omega-3 phospholipids: Metabolism and biological activities. Int. J. Mol. Sci. 2012, 13, 15401-15419. [CrossRef]

53. Alavi, M.; Karimi, N.; Safaei, M. Application of Various Types of Liposomes in Drug Delivery Systems. Adv. Pharm. Bull. 2017, 7, 3-9. [CrossRef] [PubMed] 
54. Silva AMS, A.A. Chemical Study and Biological Activity Evaluation of Two Azorean Macroalgae: Ulva rigida and Gelidium microdon. Oceanogr. Open Access 2013, 1, 1-7. [CrossRef]

55. Lee, J.-C.; Hou, M.-F.; Huang, H.-W.; Chang, F.-R.; Yeh, C.-C.; Tang, J.-Y.; Chang, H.-W. Marine algal natural products with anti-oxidative, anti-inflammatory, and anti-cancer properties. Cancer Cell Int. 2013, 13, 55. [CrossRef] [PubMed]

56. Mendes, M.; Pereira, R.; Sousa Pinto, I.; Carvalho, A.P.; Gomes, A.M. Antimicrobial activity and lipid profile of seaweed extracts from the North Portuguese Coast. Int. Food Res. J. 2013, 20, 3337-3345.

57. Arunkumar, K.; Selvapalam, N.; Rengasamy, R. The antibacterial compound sulphoglycerolipid 1-0 palmitoyl-3-0(6-sulpho- $\alpha$-quinovopyranosyl)-glycerol from Sargassum wightii Greville (Phaeophyceae). Bot. Mar. 2005, 48, 441-445. [CrossRef]

58. Ohta, K.; Mizushima, Y.; Hirata, N.; Takemura, M.; Sugawara, F.; Matsukage, A.; Yoshida, S.; Sakaguchi, K. Action of a New Mammalian DNA Polymerase Inhibitor, Sulfoquinovosyldiacylglycerol. Biol. Pharm. Bull. 1999, 22, 111-116. [CrossRef]

59. Winget, R.R. Anti-Inflammatory Compositions Containing Monogalactosyl Dieicosapentaenoyl Glycerol and Methods Relating Thereto. U.S. Patent No. 5,767,095, 16 June 1998.

60. Khansari, N.; Shakiba, Y.; Mahmoudi, M. Chronic inflammation and oxidative stress as a major cause of age-related diseases and cancer. Recent Pat. Inflamm. Allergy Drug Discov. 2009, 3, 73-80. [CrossRef]

61. Liguori, I.; Russo, G.; Curcio, F.; Bulli, G.; Aran, L.; Della-Morte, D.; Gargiulo, G.; Testa, G.; Cacciatore, F.; Bonaduce, D.; et al. Oxidative stress, aging, and diseases. Clin. Interv. Aging 2018, 13, 757-772. [CrossRef]

62. Lobo, V.; Patil, A.; Phatak, A.; Chandra, N. Free radicals, antioxidants and functional foods: Impact on human health. Pharmacogn. Rev. 2010, 4, 118-126. [CrossRef]

63. Arulselvan, P.; Fard, M.T.; Tan, W.S.; Gothai, S.; Fakurazi, S.; Norhaizan, M.E.; Kumar, S.S. Role of Antioxidants and Natural Products in Inflammation. Oxid. Med. Cell. Longev. 2016, 2016, 5276130. [CrossRef]

64. Xu, D.-P.; Li, Y.; Meng, X.; Zhou, T.; Zhou, Y.; Zheng, J.; Zhang, J.-J.; Li, H.-B. Natural Antioxidants in Foods and Medicinal Plants: Extraction, Assessment and Resources. Int. J. Mol. Sci. 2017, 18, 96. [CrossRef] [PubMed]

65. Jacobsen, C.; Sørensen, A.-D.M.; Holdt, S.L.; Akoh, C.C.; Hermund, D.B. Source, Extraction, Characterization, and Applications of Novel Antioxidants from Seaweed. Annu. Rev. Food Sci. Technol. 2019, 10, 541-568. [CrossRef] [PubMed]

66. Rey, F.; Lopes, D.; Maciel, E.; Monteiro, J.; Skjermo, J.; Funderud, J.; Raposo, D.; Domingues, P.; Calado, R.; Domingues, M.R. Polar lipid profile of Saccharina latissima, a functional food from the sea. Algal Res. 2019, 39, 101473. [CrossRef]

67. Da Costa, E.; Domingues, P.; Melo, T.; Coelho, E.; Pereira, R.; Calado, R.; Abreu, H.M.; Domingues, R.M. Lipidomic Signatures Reveal Seasonal Shifts on the Relative Abundance of High-Valued Lipids from the Brown Algae Fucus vesiculosus. Mar. Drugs 2019, 17, 335. [CrossRef] [PubMed]

68. Rico, D.; Diana, A.B.M.; Milton-Laskibar, I.; Fernández-Quintela, A.; Silván, J.M.; Rai, D.K.; Choudhary, A.; Peñas, E.; de Luis, D.A.; Martínez-Villaluenga, C. Characterization and in vitro evaluation of seaweed species as potential functional ingredients to ameliorate metabolic syndrome. J. Funct. Foods 2018, 46, 185-194. [CrossRef]

69. Leri, A.C.; Dunigan, M.R.; Wenrich, R.L.; Ravel, B. Particulate organohalogens in edible brown seaweeds. Food Chem. 2019, 272, 126-132. [CrossRef] [PubMed]

70. Silva, J.; Alves, C.; Freitas, R.; Martins, A.; Pinteus, S.; Ribeiro, J.; Gaspar, H.; Alfonso, A.; Pedrosa, R. Antioxidant and neuroprotective potential of the brown seaweed Bifurcaria bifurcata in an in vitro Parkinson's disease model. Mar. Drugs 2019, 17, 85. [CrossRef] [PubMed]

71. Yuan, Y.; Zhang, J.; Fan, J.; Clark, J.; Shen, P.; Li, Y.; Zhang, C. Microwave assisted extraction of phenolic compounds from four economic brown macroalgae species and evaluation of their antioxidant activities and inhibitory effects on $\alpha$-amylase, $\alpha$-glucosidase, pancreatic lipase and tyrosinase. Food Res. Int. 2018, 113, 288-297. [CrossRef]

72. Yuan, Y.V.; Carrington, M.F.; Walsh, N.A. Extracts from dulse (Palmaria palmata) are effective antioxidants and inhibitors of cell proliferation in vitro. Food Chem. Toxicol. 2005, 43, 1073-1081. [CrossRef]

73. Pang, J.R.; Goh, V.M.J.; Tan, C.Y.; Phang, S.M.; Wong, K.H.; Yow, Y.Y. Neuritogenic and in vitro antioxidant activities of Malaysian Gracilaria manilaensis Yamamoto \& Trono. J. Appl. Phycol. 2018, 30, 3253-3260. 
74. Lim, S.; Choi, A.H.; Kwon, M.; Joung, E.J.; Shin, T.; Lee, S.G.; Kim, N.G.; Kim, H.R. Evaluation of antioxidant activities of various solvent extract from Sargassum serratifolium and its major antioxidant components. Food Chem. 2019, 278, 178-184. [CrossRef] [PubMed]

75. Melo, T.; Marques, S.S.; Ferreira, I.; Cruz, M.T.; Domingues, P.; Segundo, M.A.; Domingues, M.R.M. New Insights into the Anti-Inflammatory and Antioxidant Properties of Nitrated Phospholipids. Lipids 2018, 53, 117-131. [CrossRef] [PubMed]

76. Magalhães, L.M.; Segundo, M.A.; Reis, S.; Lima, J.L.F.C. Automatic method for determination of total antioxidant capacity using 2,2-diphenyl-1-picrylhydrazyl assay. Anal. Chim. Acta 2006, 558, 310-318. [CrossRef]

77. Magalhães, L.M.; Barreiros, L.; Maia, M.A.; Reis, S.; Segundo, M.A. Rapid assessment of endpoint antioxidant capacity of red wines through microchemical methods using a kinetic matching approach. Talanta 2012, 97, 473-483. [CrossRef] [PubMed]

78. Ozgen, M.; Reese, R.N.; Tulio, A.Z.; Scheerens, J.C.; Miller, A.R. Modified 2,2-azino-bis-3-ethylbenzothiazoline6-sulfonic acid (ABTS) method to measure antioxidant capacity of selected small fruits and comparison to ferric reducing antioxidant power (FRAP) and 2,2'-diphenyl-1- picrylhydrazyl (DPPH) methods. J. Agric. Food Chem. 2006, 54, 1151-1157. [CrossRef] [PubMed]

(C) 2019 by the authors. Licensee MDPI, Basel, Switzerland. This article is an open access article distributed under the terms and conditions of the Creative Commons Attribution (CC BY) license (http://creativecommons.org/licenses/by/4.0/). 